\title{
Lo sfondo storico-iconografico dell'immagine di Cristo nel Tempietto Longobardo di Cividale
}

\author{
Alla memoria dell'amico Anton Marcus Hassel \\ morto nel campo di concentramento \\ di Sachsenhausen il 26 aprile 1944
}

\begin{abstract}
The wall-paintings in the Tempietto Longobardo at Cividale, ca 750/760, are rendered in an unmistakable Byzantine style. They were probably made by a workshop lead by a master painter from Constantinople. As such, the paintings are important as rare evidence of figural religious art from the iconoclastic period. The technically most accomplished part of the decoration is the painting depicting Christ flanked by the archangels Michael and Gabriel in the lunette over the western door, the main entrance to the chapel. The very distinctive physiognomic type of the Christ indicates that the painter rendered a specific prototype. The article discusses parallels and traces the origin of this particular iconographic type.

Il Tempietto di Cividale del Friuli è un monumento chiave della storia dell'arte dell'alto medioevo - tanto del Mediterraneo, quanto d'Oltralpe, tanto dell'Occidente, quanto dell'Oriente. È questa importanza storica generale del monumento che qui vorrei sottolineare partendo dagli affreschi del primo strato. ${ }^{1}$ Insieme agli stucchi, queste pitture fanno parte della decorazione originaria del monumento. ${ }^{2}$ La datazione dell'architettura e delle decorazioni intorno alla metà dell'ottavo secolo, cioè agli ultimi decenni del Regno longobardo, oggi è generalmente accettata e non richiede ulteriori commenti. ${ }^{3}$ Occorre, d'altra parte, mettere in rilievo il fatto che all'origine, cioè quando venne progettato, costruito e decorato, il Tempietto faceva parte della gastaldaga, la sede del gastaldius regis, il rappresentante del re longobardo. Siccome questa sede de facto e de jure
\end{abstract}

1. L'articolo sviluppa idee già suggerite in altri scritti, recentemente nel libretto pubblicato dal Municipio di Cividale del Friuli (Torp 2006).

2. Torp 1953, 83; Torp 2006, 24

3. Per le varie tappe nella datazione degli affreschi, si veda Torp 1959, 6-11; Torp 2009. Per tentativi di ri-lettura delle fasi edilizie del Tempietto, si veda Casirani, Cernuschi, Codini 2002, 107-159, fig. 1-9; Lusuardi Siena 2002, 205-250, fig. 1-8 (non trovo convincenti tutte le congetture presentate ma, nell'attuale contesto, non trovo necessario discuterle). 


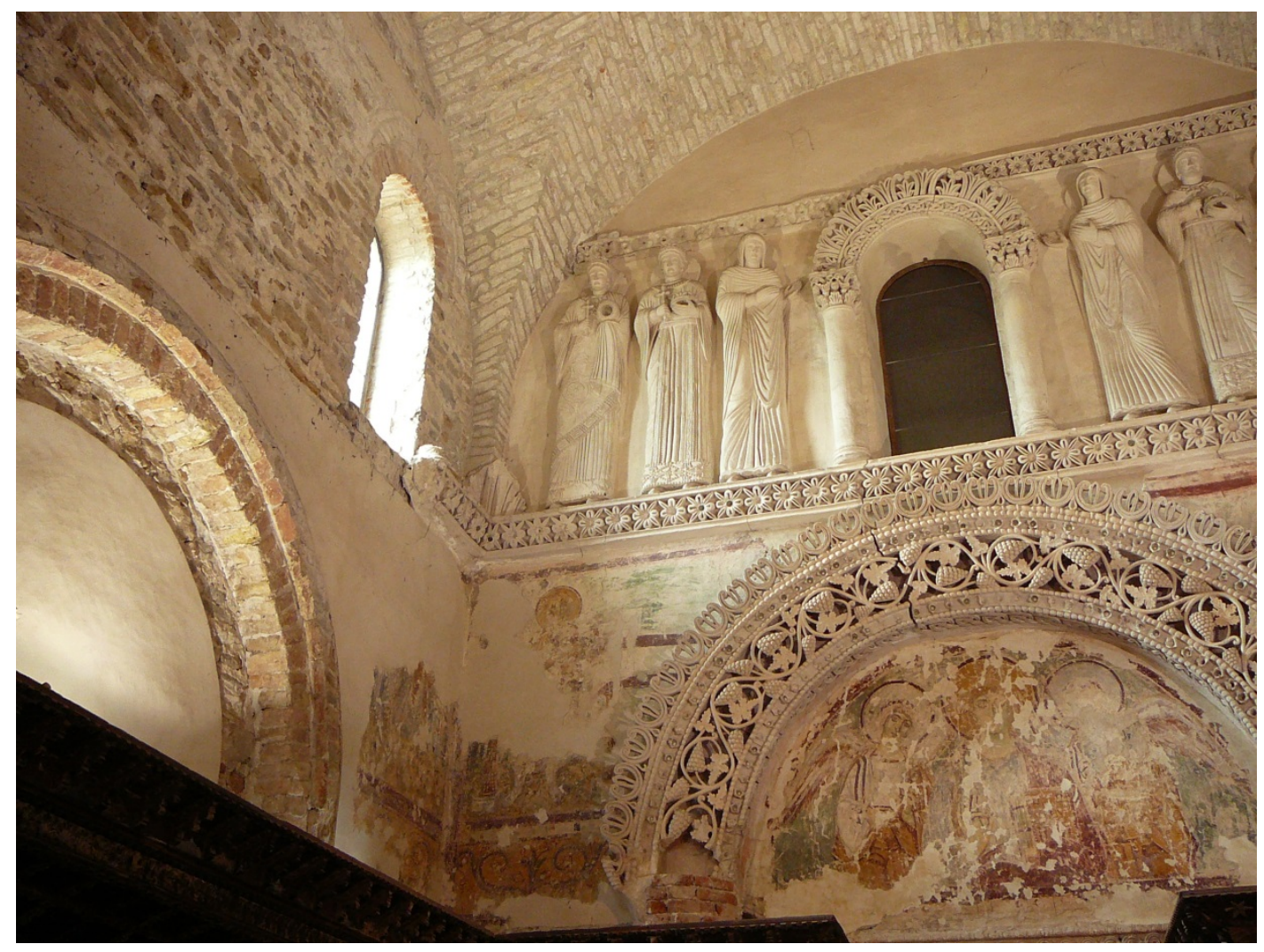

FIG. 1 - Cividale, Tempietto Longobardo, le pareti sud ed ovest, visti dal basso (photo: B. Kiilerich).

era sottomessa alla giurisdizione del re, residente a Pavia, il Tempietto, sito nella locale curtis regia, era una cappella palatina. ${ }^{4}$

La cappella consiste di due corpi architettonici semplici e ben distinti: a ovest si trova l'aula, a est il presbiterio. Quest'ultimo è basso, e consta di tre vani paralleli coperti da volte a botte sorrette da blocchi di trabeazione supportati da colonne, mensole e pilastri. La parete di fondo del vano centrale è messa in risalto da un arcone cieco, poco profondo; la porta d'accesso al presbiterio si apre a sud. L'aula, alta e di struttura cubica è coperta da una volta a crociera (in larga parte ricostruita) $;^{5}$ i tre arconi ciechi dei muri perimetrali sono alti, ampi e profondi; l'entrata principale, situata nell'arcone ovest, collegava il sacello all'atrio del complesso residenziale della gastaldaga. $^{6}$

Le parti più importanti delle ricche decorazioni originarie conservate si trovano nell'aula (FIG. 1). Suddivise in zone orizzontali, queste erano originariamente composte di lastre marmoree, di semicolonne, archi, fregi e grandi figure di stucco, di affreschi e, nella volta, di mosaici a fondo

4. Torp 1977, 227-229. Cf. Casirani 2002, 61-76, fig. 1-35.

5. Torp 1977, 12-16.

6. Nella fase iniziale l'edificio era privo sia di nartece o atrio (a ovest) che di sagrestia (a sud), cf. Torp 1977, 54-
59; 62-66. Per l'aspetto originale della facciata occidentale, si veda Degani $1990^{2}$, 22-23 (fig.), 68-73 (fig. di dettagli della fronte; per i valori proporzionali della costruzione, Tavano 1990, 51-59. 


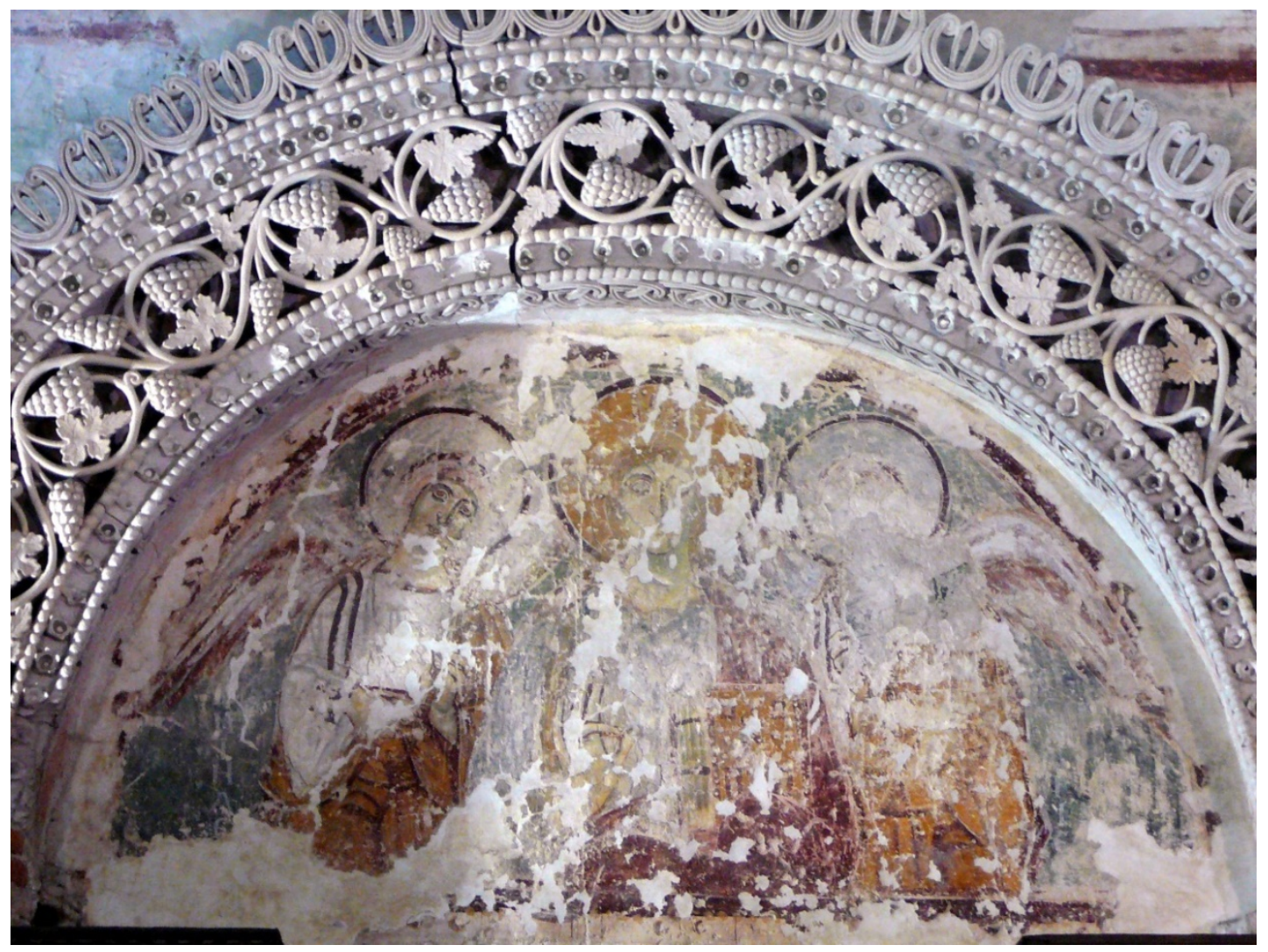

FIG. 2 - Cividale, Tempietto Longobardo, parete ovest: lunetta con l'affresco di Cristo fra gli arcangeli Michele e Gabriele (photo: B. Kiilerich).

oro. Così come la struttura architettonica, anche le zone e i singoli elementi delle decorazioni erano coerentemente proporzionati e uniti per mezzo di un sistema artistico d'integrazione, elaborato a Bisanzio. ${ }^{7}$ La presente relazione si limita a considerazioni relative alla zona degli affreschi delle pareti nord, ovest et sud, zona che si sviluppa tra il rivestimento di marmo (oggi rimosso) e le figure di stucco. ${ }^{8}$ Sopra la porta principale è rappresentato il Cristo (FIG. 2), nella lunetta dell'arcone nord e in ciascuno dei campi laterali dei tre arconi sono conservate parti importanti di affreschi, rispettivamente della Vergine (FIG. 3) e di sei santi (FIG. 4). Sotto i quattro santi a ovest è inserito un corposo tralcio d'acanto, mentre sotto i due santi a est e sulle pareti del presbiterio (interrotto solo dall'arcone cieco di levante) è inserita una fascia con una iscrizione di tre righe: l'iscrizione dedicatoria che, secondo il modello imperiale, è dipinta di bianco su sfondo porpora e (come le immagini dipinte) inquadrata in una cornice di gemme colorate e perle bianche (FIG. 5). ${ }^{9}$

7. Torp 1984, 92-105, figg. 30, 32, 33a; Torp 2006, 24-26, dis. I-III (28-30).

2008; Kiilerich 2010.

8. Rispetto agli stucchi, mi limito a citare qualche contributo tra i più recenti, van Lohuizen-Mulder 1990; Casadio, Perusini, Spadrea 1996; Vaj 2002; Kiilerich

9. I miei calchi delle iscrizioni dipinte sono riprodotti da L'Orange 1953, tav. XIIa-c. Cf. Torp 1959, $27-$ 29, figg. 16-19; Torp 1974, 10-12, tavv. 15.2, $16.1,2$. 


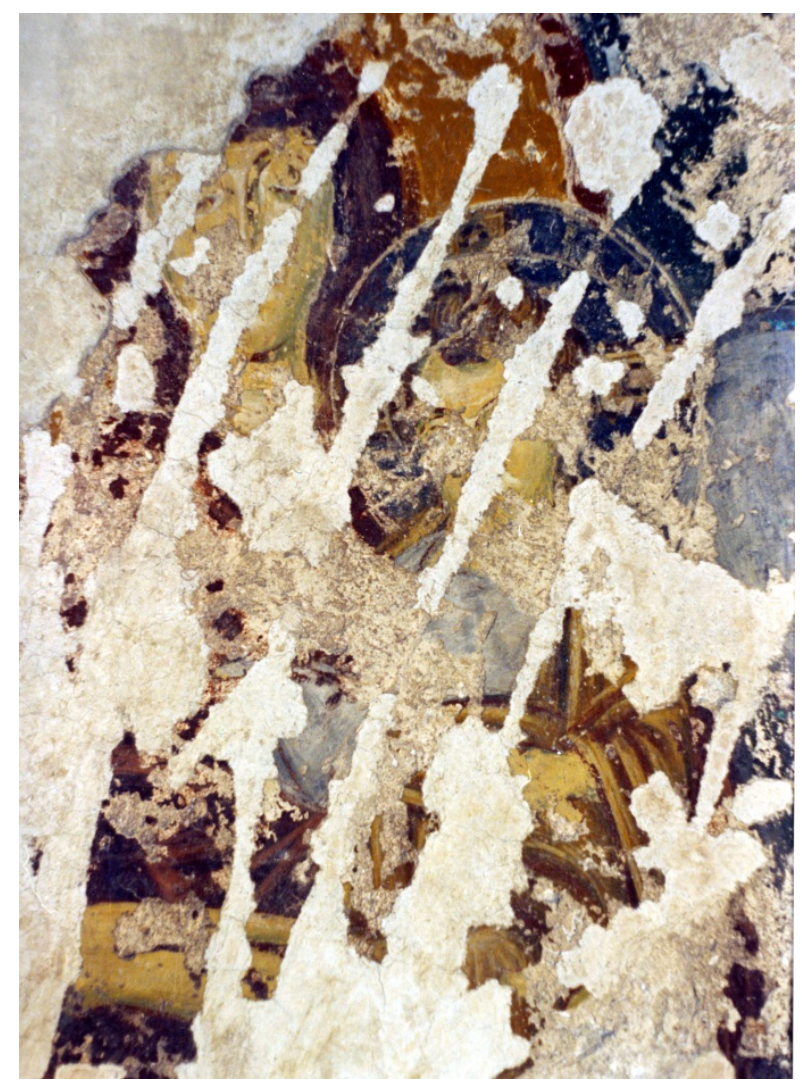

FIG. 3 - Cividale, Tempietto Longobardo, parete nord: particolare della Vergine Hodighitria con il Bambino (photo: H. Torp).

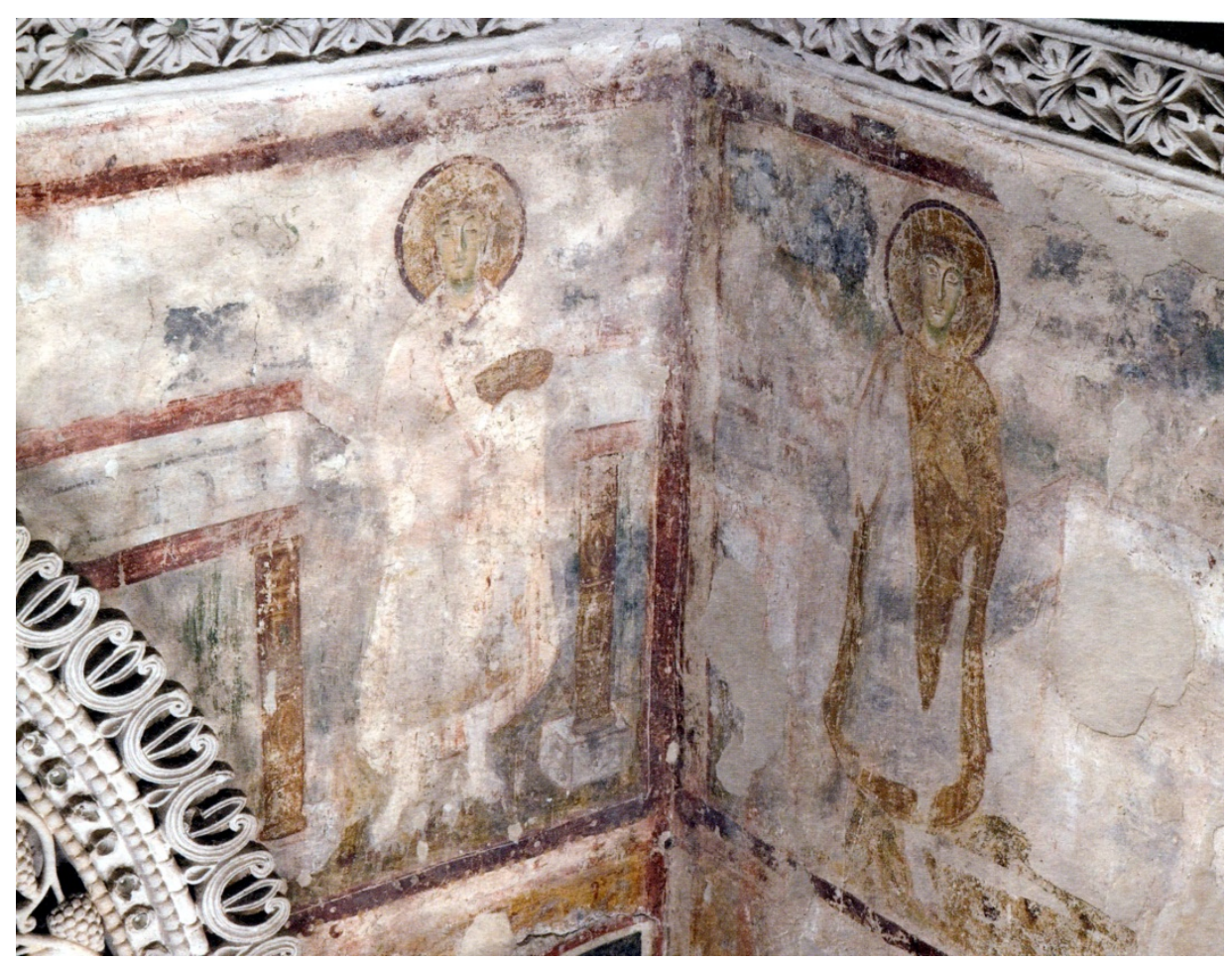

FIG. 4 - Cividale, Tempietto Longobardo, angolo NO dell'aula con due martiri militari (da: Torp 2006, tav.17). 


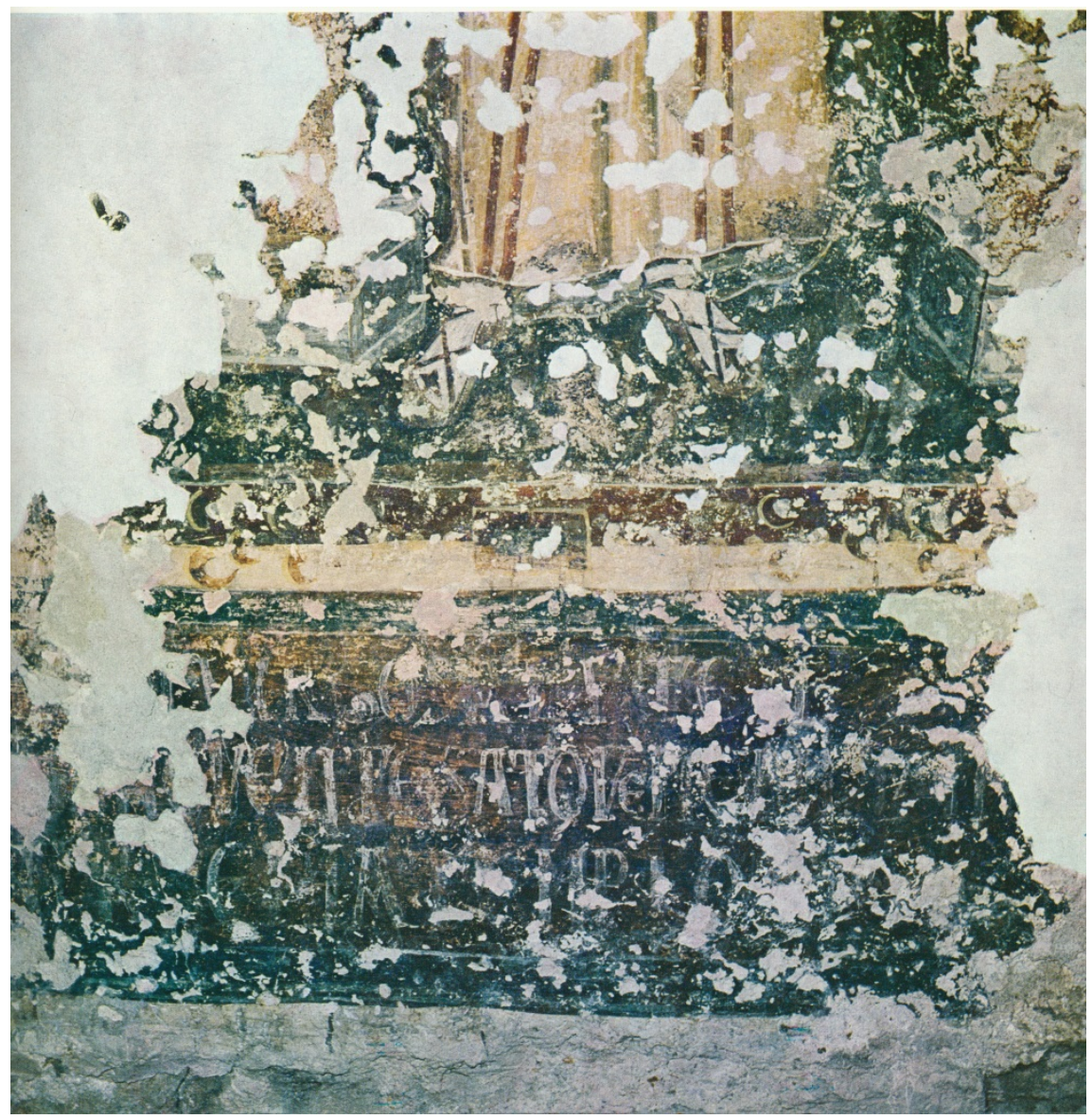

FIG. 5 - Cividale, Tempietto Longobardo, pennacchio sud-est dell'aula: frammento.

I nomi dei PIOS AVCTORES non sono più leggibili, ma tutto fa pensare che i committenti della cappella palatina furono Giseltrude e Astolfo, coppia regnante nell'infausto periodo tra il 749 e il 756.

Nella prima decorazione di affreschi, il legame del sacello con la gastaldaga emerge chiaramente. A questo riguardo è illuminante il fatto che dei sei santi rappresentati in piedi ai lati dei tre arconi dell'aula, l'unico ecclesiastico è il vescovo (leggermente più alto di statura) rappresentato nel pennacchio sud-est, vicino alla porta d'accesso del presbiterio. In ognuno degli altri cinque pennacchi, invece, figura un personaggio vestito di tunica e clamide militare: sono santi guerrieri.

Disposti frontalmente, i tre martiri hanno grandi occhi, aperti e fissi, e la testa circondata da un nimbo giallo scuro orlato di porpora. La forma ovale, finemente modellata dei volti, il dorso nasale lungo con la punta trilobata, le palpebre curve dipinte in rosso scuro, verde e bianco, le sopracciglia scure tendenti a unirsi sopra la radice del naso, la disposizione geometricamente regolare delle luci, sono tutte caratteristiche che si ripetono di volto in volto e in generale rimandano ad un modello bizantino (FIG. 6, 7), o, per l'altissima qualità della testa FIG. 8, ad una mano bizantina. ${ }^{10}$

10. Torp 1959, 8-11, figg. 3-5. 


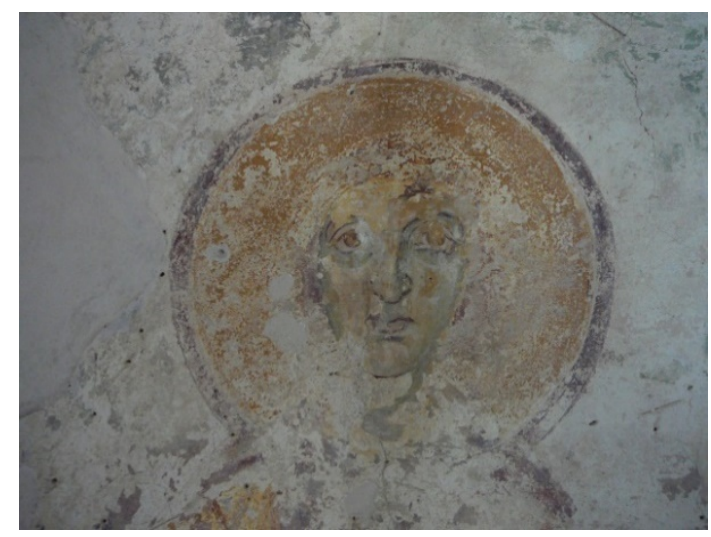

FIG. 6 - Cividale, Tempietto Longobardo, volto di santo militare, parete ovest (cf. fig. 1) (photo: B. Kiilerich).

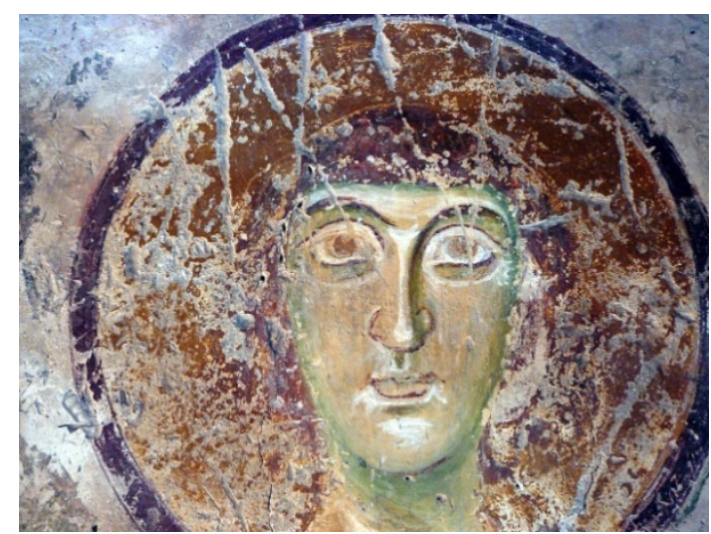

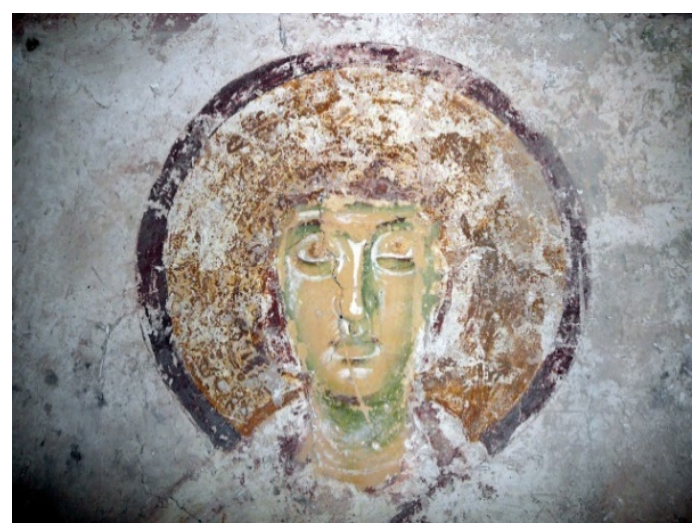

FIG. 7 - Cividale, Tempietto Longobardo, volto di santo militare, parete ovest (cf. fig. 4) (photo: B. Kiilerich).

La massa dei capelli bruni scuro è tagliata con una acconciatura alla paggio. Con delle pennelate sottili, oggi largamente scolorite, parzialmente scialbe e appena visibili sono dipinti dei riccioli che dall'alto si dirigono verso la bassa fronte, dove il contorno leggermente ondulato suggerisce una fila di Buckellokken (FIG. 9).

Il gastaldius regis aveva obblighi militari, ma l'iconografia riflette verosimilmente anche il legame diretto del Tempietto con le autorità centrali. Il santo dipinto a sinistra dell'arcone nord (FIG. 4,8 ) è l'unico a cui possa essere attribuito con qualche certezza il nome: [SANCTUS HAD] REANUS, soldato martire al quale il re Ansprando (712) aveva dedicato un apposito santuario nella necropoli longobarda fuori Pavia, la città capitale dei Longobardi. ${ }^{11}$ Visto che le decorazioni del Tempietto cividalese vennero eseguite in un momento storico estremamente precario per il Regno, è naturale che fu data particolare importanza ai martiri militari come intercessori presso le potenze divine. ${ }^{12}$

11. Torp 1977, 239

12. Cf. Torp 2011, 40-41, nei mosaici della Rotonda di Tessalonica, "originally, nine of the twenty saints may have been soldiers. Reinforced by ecclesiastics and laymen, the unit of soldier martyrs has been allotted the lead in defending the Empire (...). By emphasising holy warriors, the imperial commissioner and his learned advisors have appropriated - or perhaps even invented? - a specific iconographic programme for palatine churches. This programme emphasising military saints is documented later by the wall-paintings in the so-called Tempietto Longobardo (...) and still later by the mosaics in the Norman kings' Cappella Palatina at Palermo (...)." 


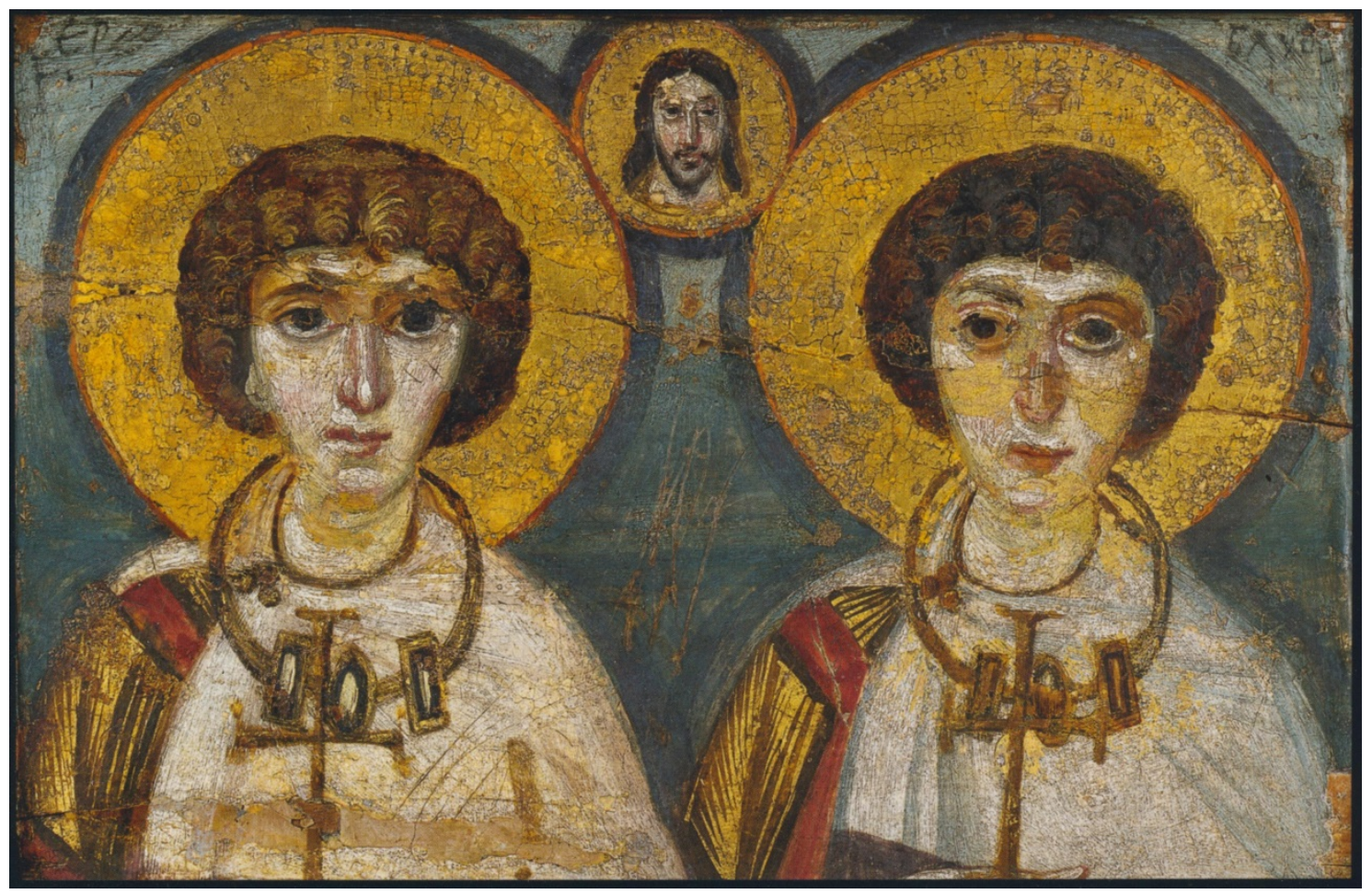

FIG. 9 - Icona con Sergio e Bacco, Kiev, Bohdan e Varvara Khanenko Museo (da: Nelson \& Collins 2006, p. 126).

Colpisce il fatto che i tre ritratti conservati dei martiri militari si somigliano tanto da apparire, per così dire, fisionomicamente uniformi. Le differenze sono dovute principalmente al diverso stato di conservazione e al fatto che, con ogni probabilità, i ritratti sono opere di mani diverse. ${ }^{13} \mathrm{Si}$ può senz'altro concludere che questi militari, adolescenti e glabri, furono dipinti secondo un ben definito modello base: il giovane idealizzato, "spogliato dell'uomo vecchio" (S. Paolo, Col 3, 10) ed elevato al di sopra della sfera terrestre. ${ }^{14}$ Originariamente, si vedeva probabilmente un coro di cinque soldati martiri uniformi, fatto sorprendente, dato che, nell'arte paleocristiana, le immagini dei santi e delle sante traggono origine da ritratti individualizzati.

Conformemente alla tradizione paleocristiana, sono individualizzati per esempio i ritratti dei santi militari Sergio e Bacco dipinti in Santa Maria Antiqua a Roma probabilmente nel tempo di papa Paolo I (757-767): Bacco d'aspetto giovanile, imberbe e dai capelli biondi, Sergio ritratto con piccoli baffi e capelli scuri. ${ }^{15}$ Il culto di Sergio e Bacco, e con esso le loro reliquie e immagini, furono trasmesse da Bisanzio a Roma nel VI secolo. ${ }^{16}$ Di conseguenza, anche l'iconografia individualizzante dei due militari è di probabile origine costantinopolitana. Eppure, nella celebre icona di Sergio e Bacco conservata nel Museo Bohdan e Varvara Khanenko di Kiev (FIG. 9), i due santi sono dipinti in base alla iconografia "gemellare" dei martiri militari del Tempietto. ${ }^{17}$

13. A Brescia, S. Salvatore, contemporaneo ritratto di santo, analogo ma non identico, Torp 1959, fig. 15a; Hubert et al. 1967, fig. 136 (121).

14. Per la bibliografia, si veda Kiilerich 2007, 328, n. 15.

15. de Grüneisen 1911, tavv. XXVIII-XXIX.

16. Rushforth 1902, 31.

17. Nelson, Collins 2006, 127, pl. a colori (per la datazione, piuttosto che il sec. VII inoltrato, gli autori preferiscono il periodo di Giustiniano I). 

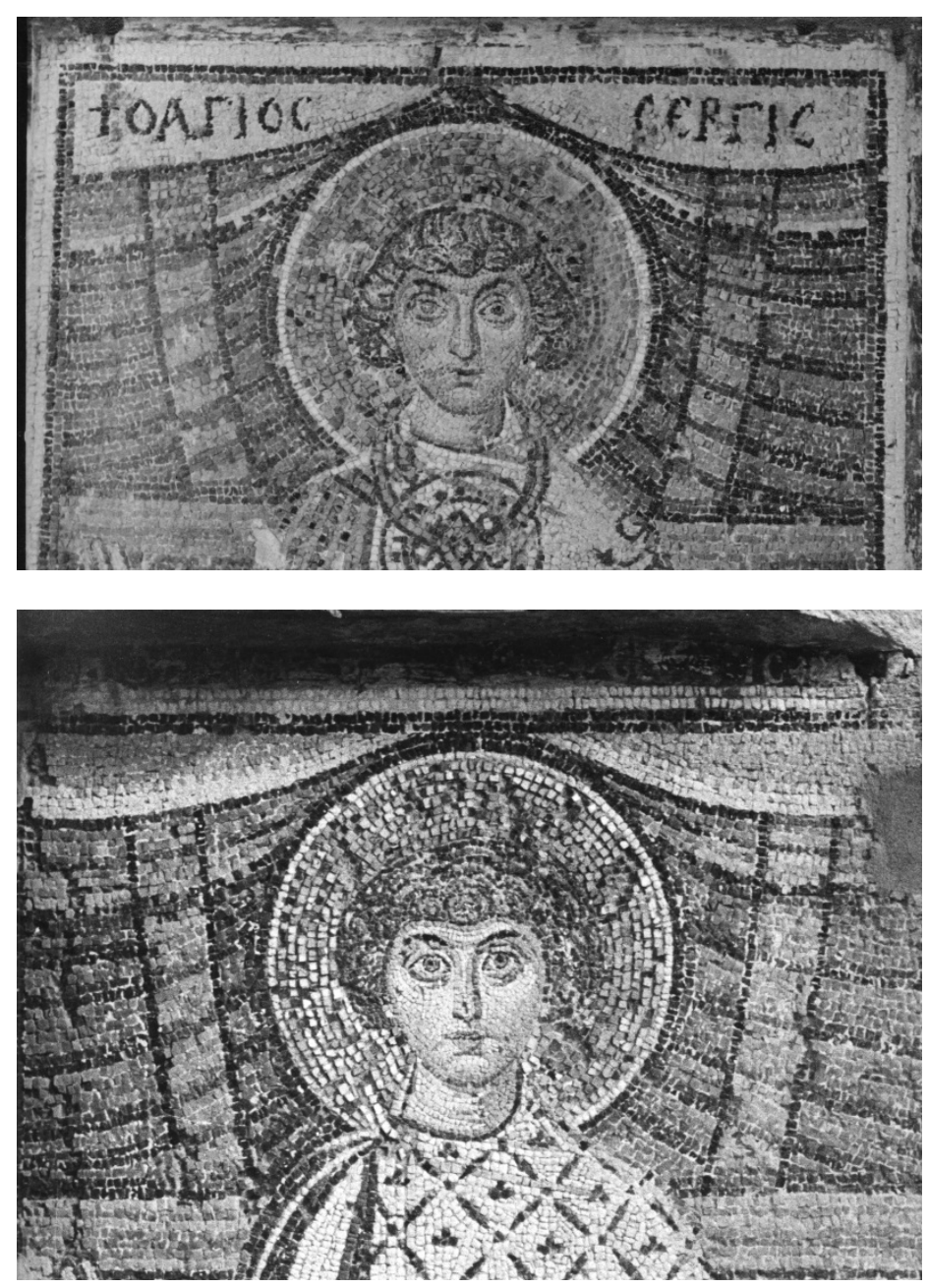

FIg. 10 - Tessalonica, Basilica di S. Demetrio, mosaico di S. Sergio (photo: H. Torp).

FIG. 11 - Tessalonica, Basilica di S. Demetrio, mosaico di S. Giorgio (photo: $\mathrm{H}$. Torp).

Trovata nel monastero del Sinai, ma eseguita probabilmente a Costantinopoli nel secolo VII, l'icona suggerisce che anche l'iconografia "gemellare" dei martiri militari sia stata sviluppata nella metropoli. ${ }^{18}$ Verosimilmente destinata a esprimere il concetto dell "essere celeste", l'iconografia "gemellare" con ogni probabilità è stata sviluppata come parallelo "teologico" alla tradizione paleocristiana individualizzante.

Un'immagine del tipo di Sergio e Bacco dell'icona e dei militari dipinti nel Tempietto è offerta dal bellissimo mosaico del primo terzo del VII secolo di Sergio sulla faccia ovest del pilastro destro all'ingresso del coro della basilica di San Demetrio a Tessalonica (FIG. 10). Sul lato opposto dell'ingresso, il riquadro corrispondente ci presenta San Giorgio (FIG. 11), raffigurato secondo la stessa iconografia idealizzante "gemellare" - mentre nei coevi mosaici il santo titolare Demetrio è ritratto con poca variazione, secondo la locale iconografia tradizionale. ${ }^{19}$

18. Cf. Torp 2014, 277, fig. 2 (268), ritratto del martire Leone, Rotonda di Tessalonica, opera che penso sia fatta prima della fine del IV sec.
19. Bakirtzis, Kourkoutidou-Nikolaidou, Mavropoulou Tsioumi 2012, 158, 164, 166, figg. 36, 39 (Giorgio), $43,45,46$ (Sergio). 
Il nuovo modo "spirituale" di presentare i santi militari è stato verosimilmente trasmesso da Bisanzio dal maestro incaricato dalla corte longobarda di preparare e dirigere il complesso progetto decorativo del Tempietto. ${ }^{20} \mathrm{Il}$ rapporto con l'arte e l'iconografia di Bisanzio si afferma negli affreschi delle lunette degli arconi settentrionale e occidentale. ${ }^{21}$ Nonostante i grandi danni, è chiaro che la lunetta nord offriva a chi pregava l'immagine di (SCA) MA(R)IA (FIG. 3), affiancata dagli arcangeli Michele e Gabriele, ambedue con uno scettro coronato con una globetta e ornato di perle nella mano destra e con una grande sfera trasparente nella mano sinistra. ${ }^{22}$ In piedi (sicuramente su un suppedaneo) con il Bambino sul braccio sinistro, la Vergine è del tipo noto sotto il nome di Hodighitria. ${ }^{23}$ Il nimbo di Maria è di color giallo, quello del Figlio, crucifero e blu scuro, quello degli angeli di un blu-grigio tenente all'argento. È sorprendente la parentela iconografica dell'affresco di Cividale con il mosaico absidale della piccola chiesa della Panaghia Angeloktistos a Kiti in Cipro (VII secolo): oltre allo schema generale e agli attributi (scettro, sfera), si notano particolarmente i nimbi d'argento degli arcangeli. ${ }^{24}$

Come testimoniata da sigilli bizantini, l'immagine della Hodighitria (di solito col Bambino proprio sul braccio sinistro) negli ultimi decenni del settimo secolo e nei primi del ottavo rappresentava l'icona-palladium imperiale. Nel 717, sotto Leone III l'Isaurico (717-741), il futuro iniziatore dell'iconoclastia, la terza vittoriosa difesa di Costantinopoli contro i Musulmani fu legata all'intervento miracoloso della Madre di Dio, presente nell'icona portata in processione sulle mura del capitale durante l'assedio. ${ }^{25}$ Dato che anche questo imperatore aveva scelto per i propri sigilli l'immagine della Hodighitria, è lecito pensare che l'immagine della Vergine con il Figlio che pochi decenni dopo venne dipinta nella lunetta settentrionale della cappella del gastaldius regis fosse ispirata dalla vittoriosa icona bizantina. ${ }^{26}$ Però, neppure il palladium mariano potè salvare il regno dei Longobardi dell'Italia settentrionale, stretto tra il papa di Roma e il re dei Franchi.

La presenza artistica e culturale del Bisanzio imperiale si manifesta in modo ancor più singolare e significativo dalla splendida icona di Cristo dipinta nella lunetta occidentale, cioè nella controfacciata, sopra la porta che connette la cappella con il palazzo (FIG. 2). Come la Madre della lunetta nord, il Figlio è affiancato dagli arcangeli Michele e Gabriele che, con la mano più vicino al Cristo, reggono uno scettro dello stesso tipo raffigurato nella lunetta nord, con l'altra sostengono davanti a sé un lembo del pallio.

20. Torp 2006.

21. Nella lunetta meridionale non rimane quasi nulla dell'affresco originario, ma immaginando che vi fosse l'immagine di Giovanni Battista (affiancato da Elisabetta e Zaccharia), le tre lunette dell'aula avrebbero accennato alla rappresentazione simbolica (dal secolo XI) chiamata Deesis, scena di intercessione documentata da un affresco della metà del VII secolo in S. Maria Antiqua, Nordhagen 2001, 324, fig. 5; comportante un donatore, l'iconografia potrebbe riflettere "an imperial usage"; cf. Nordhagen 2004, 208, "ruler-instigated".

22. Torp 1999, 583-587. Le sfere sono prive della solita crocetta, presente per esempio nel mosaico di Kiti, Chatzidakis 1994, pls. 21, 22. Per gli scettri, cf. 8, n. 49. Per la bibliografia, si veda Kiilerich 2007, 328, n. 15.

23. L'epiteto rimonta probabilmente solo al secolo XI ma, come documentano l'affresco cividalese e, come vedremo, dei sigilli bizantini, il tipo iconografico è molto più antico; cf. Torp 1999, 589-593.
24. Ibid., 587, n. 11-13. Per gli scettri degli angeli, si veda infra, 94, n. 53.

25. Per la documentazione, si veda Torp 1999, 590-593. Secondo L. Brubaker 2012, 27-29, l'iniziatore dell'iconoclastia fu non Leone, ma suo figlio Costantino V, imperatore assoluto 741-775, che "appears as an iconoclast in the late 740 s or early $750 \mathrm{~s}$ " (33).

26. Dal punto di vista iconografico, l'icona della Hodighitria ora nel Pantheon (Bertelli 1961; Aurea Roma, no. 376, 661-662) è vicina all'affresco del tempietto; formalmente, però, le due immagini sono molto dissimili. Per la complessa cultura artistica altomedievale a Roma, si veda Pace, 2004, 147-149; Pace 2015. Per considerazioni sulla iconografia a Roma della Vergine con il Bambino sul braccio (Hodighitria e Blacherniotissa), cf. anche Weis 1958, 32-38. 


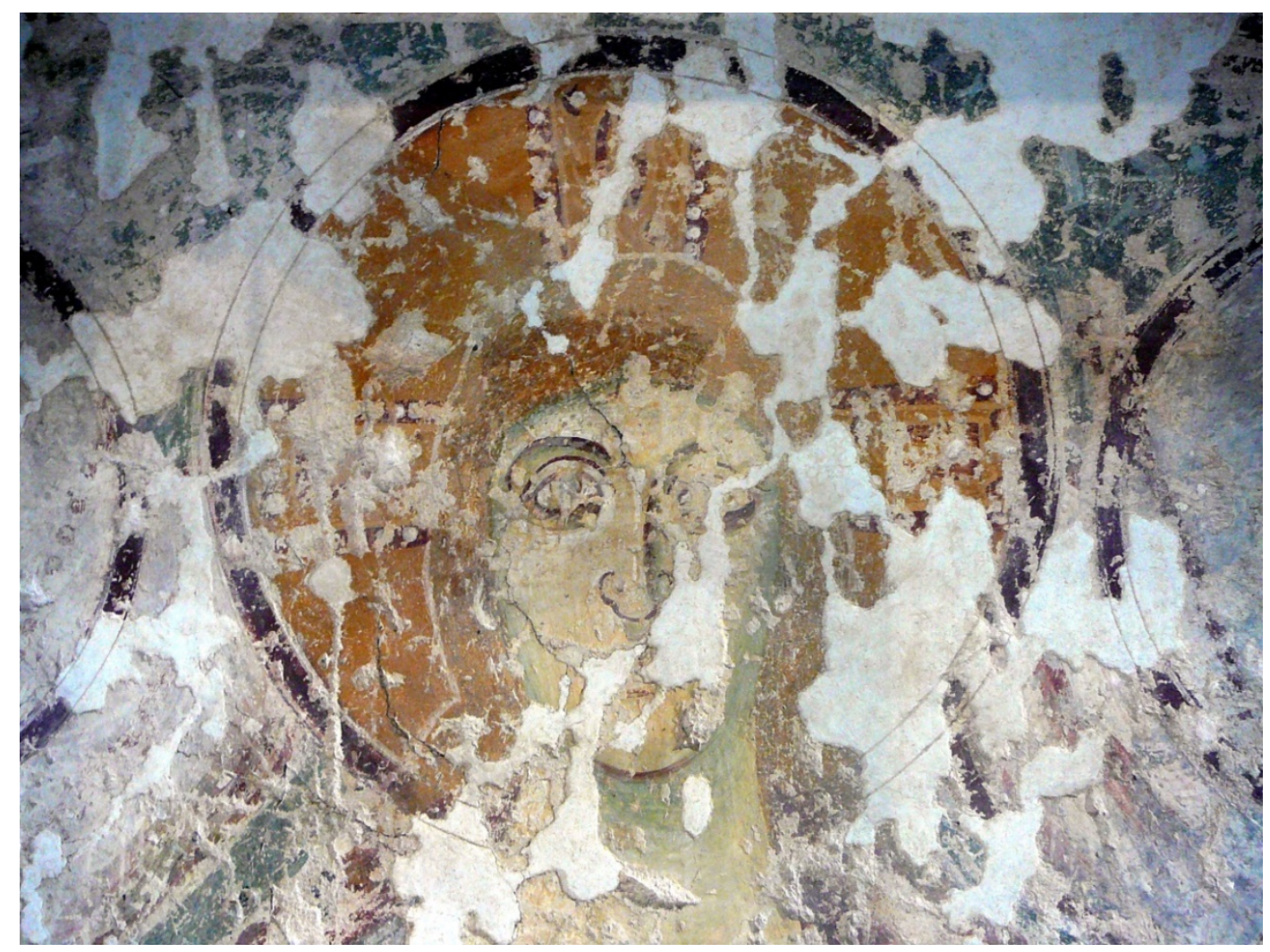

FIG. 12 - Tempietto Longobardo, Cividale, volto di Cristo (cf. fig. 2) (photo: B. Kiilerich).

L'immagine del Cristo (FIG. 12) costituisce la componente non solo di più alta qualità tecnica ed estetica del ciclo pittorico, ma è anche l'elemento più carico di significato di tutte le figurazioni conservate nel Tempietto. Ai due lati del nimbo crociato si legge il monogramma grecolatinizzato del nome, oggi parzialmente distrutto: IHS XPS. I capelli lunghi e biondi del Salvatore hanno la scriminatura in mezzo e, cadendo sulle spalle, definiscono la forma ovoidale e pressoché scultoreamente modellata del viso imberbe. Alcune ciocche pendono sulla fronte larga e bassa; gli occhi, grandi e spalancati, le curve delle palpebre, dei cigli e dei sopraccigli occupano gran parte della bassa fronte; il naso è lungo e stretto; la parte inferiore della faccia e il collo, alto e largo, sono di pesantezza quasi eccessiva.

A Cividale, una rappresentazione di Cristo dello stesso tipo fisionomico - ma realizzato in un diverso linguaggio figurativo - domina la lastra frontale di pietra calcarea, originariamente dipinta, dell'altare del duca Ratchis (737-744). ${ }^{27}$ Anche se formalmente molto differenti, i numerosi adattamenti del tipo fisionomico di Cristo, in scultura, pittura o in disegno colorato, documentano la sua larghissima diffusione nell'Alto Medievo. ${ }^{28}$ Particolarmente interessante è una delle due rappresentazioni del "tipo cividalese" scolpite sul sarcofago merovingio attribuito all'episcopo

27. Chinellato 2010, fig. 62; cf. fig. 417 (ricostruzione della policromia).
28. Per una scelta rappresentativa si veda Hubert et al. 1967, figg. 150, 192, 197, 210, 212, 214, 313. 
Agilberto (ca 680) nella "Crypte de Saint-Paul" a Jouarre: nel rilievo del lato sinistro, i quattro Viventi accompagnano Cristo voltando la testa e lo sguardo, non verso Lui, ma verso l'esterno della composizione.$^{29}$ Particolare iconografico che, secondo J. Hubert, "non si vede che in un mosaico paleocristiano di Tessalonica (...)", cioè nel mosaico absidale di Hosios David. ${ }^{30}$

La predilezione delle alte sfere sociali per il tipo di Cristo del Tempietto è confermato dall'Evangeliario di Godescalco, eseguito tra gli anni 781-783 per ordine di Carlo Magno e della regina Ildegarda: non solo lo schema iconografico generale, la fisionomia e i capelli, ma anche dettagli come la croce gemmata del nimbo, i colori della tunica e del pallio, come pure l'iscrizione IHS XPS, rispecchiano un modello vicinissimo all'affresco. ${ }^{31}$ Altre tre miniature dell'epoca carolingia, poi, ci forniscono una prima indicazione del particolare senso teologico attribuito all'immagine di Cristo della lunetta ovest. Nella prima pittura, appartenente a un codice scritto probabilmente a Nonantola intorno all'anno 800, il Cristo in trono non solo è accompagnato dalla iscrizione REX REGUM ma la croce del suo nimbo è ornata - invece che da gemme dalle lettere $\mathrm{L} \mathrm{V} \mathrm{X}{ }^{32}$ Gli altri due manoscritti, illuminati nello scriptorium di Tours sotto l'abate Fridugiso (807-834), lo mostrano accompagnato dai quattro Viventi "riposante nelle regioni eteree al disopra della volta celeste" - di nuovo come nel mosaico absidale di Hosios David. Oltre alle didascalie Hac sedet arce ds mundi rex gloria caeli, rispettivamente Hic mundi caelique sedet Rex summus et auctor, il senso particolare del giovane Redentore è esplicitamente segnalato, in una delle miniature dalle parole iscritte sui bracci della croce del nimbo: REX LEX LVX ${ }^{33}$, nell'altra da due parole dette da Gesù stesso $(\mathrm{Gv} 8,12)$, iscritte sulle pagine del Vangelo aperto che sorregge: $\Phi \Omega C \mathrm{C} \Omega \mathrm{H}$, LUCE VITA ${ }^{34}$.

Tralasciando le versioni più o meno attenuate della fisionomia del Cristo cividalese fornite dai mosaici della cappella di S. Aquilino a Milano, del mausoleo di Galla Placidia a Ravenna - due fondazioni imperiali - e della chiesetta di Hosios David a Thessalonica, mi limito a citare il Salvatore sulla formella con la scena della Parusia della porta lignea di S. Sabina a Roma (422-432), rilievo che per lo stile ci riporta alla Costantinopoli di epoca paleocristiana. ${ }^{35}$

Ad eccezione dell'acconciatura, del tutto consueta, la fisionomia del Salvatore dipinto nella lunetta occidentale è singolare. Delineata con fermezza e precisione calligrafiche, l'immagine fa capire che il maestro pittore ha riprodotto un modello specifico, e che l'ha fatto ripetutamente. Come già ho accennato nel libretto sul Tempietto, pubblicato nel 2006 dal Comune di Cividale, siamo qui di fronte all'adattamento cristiano della sacra formula del ritratto di Costantino e dei

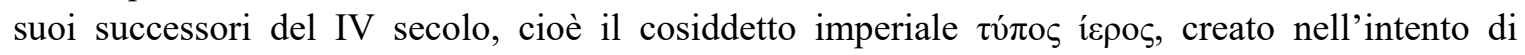
esprimere visualmente la natura o l'essenza superumana, anzi, divina, dell'imperatore. Due esempi del "tipo santo" imperiale ci sono forniti dal piccolo ritratto di Costanzo II (?) inciso su un piatto d'argento conservato nel museo dell'Ermitage di San Pietroburgo (Inv. 1820/79. FIG. 13) , $^{36}$ e

29. Hubert et al. 1967, 72-78, fig. 89 (da calco).

30. Ibid., 78 (Hubert). Cf. Elbern 1971, 16-20.

31. Paris, Bibliothèque Nationale, Ms. nouv. acq. lat. 1203, fol. 3r. Torp 1953, 12-14, tav. X.

32. Vercelli, Biblioteca Capitolare, codice CXLVIII (Omelie di san Gregorio), fol. 8r; Gabrielli 1953, 305 ("ultimo quarto VIII s.”), pl. CXLIX; Hubert et al. 1967, fig. 163; Torp 2006, fig. 1.

33. Berlin, Staatsbibl. Lat. Theol. Fol. 733 (Evangeliario), fol. $17^{\mathrm{v}}$, Koehler 1933, pl. 93c.
34. Stuttgart, Landesbibl. II, 40 (Evangeliario), fol. $1^{\mathrm{v}}$; Koehler 1933, 40-245, pl. 20a.Cf. Belting 1968, 39 (pitture nella cripta della chiesa carolingia al Volturno), figg. 39, 41, Crocifissione, nimbo del Cristo barbuto (!) con inscritta $\Phi \Omega C$; figg. 45,47 , Cristo giovane, glabro, con identico nimbo.

35. Jeremias 1980, 83-85, 99, 103-104; pls. 68-69.

36. Delbrueck 1933, 147-151, fig. 45; pl. 57. 


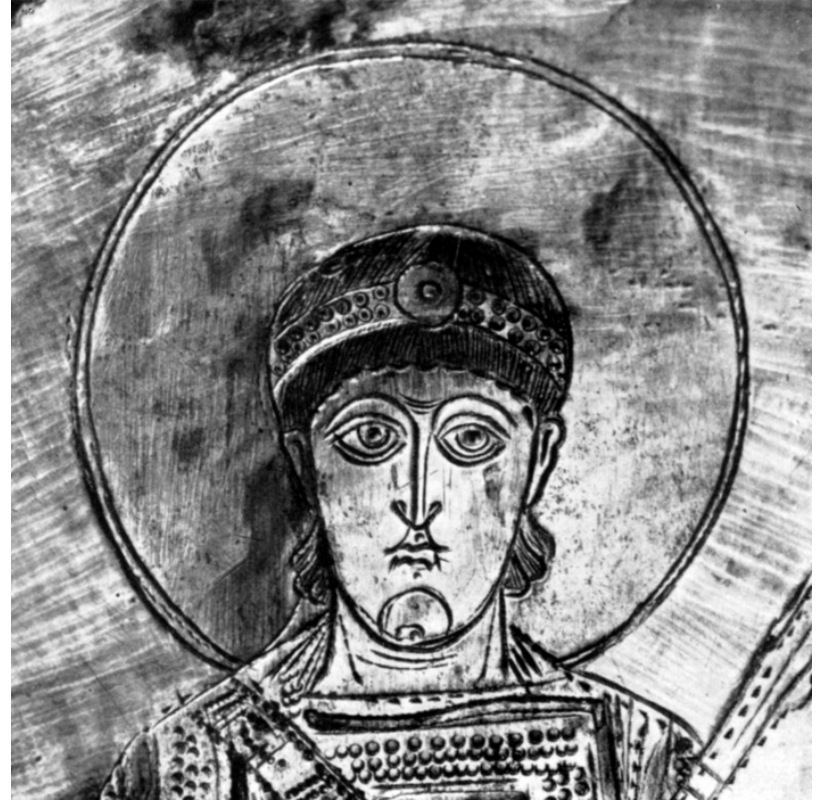

FIG. 13 - Ritratto di Costanzo II (?), piatto d'argento, San Pietroburgo, (da: Delbrueck 1933, Abb. 45).

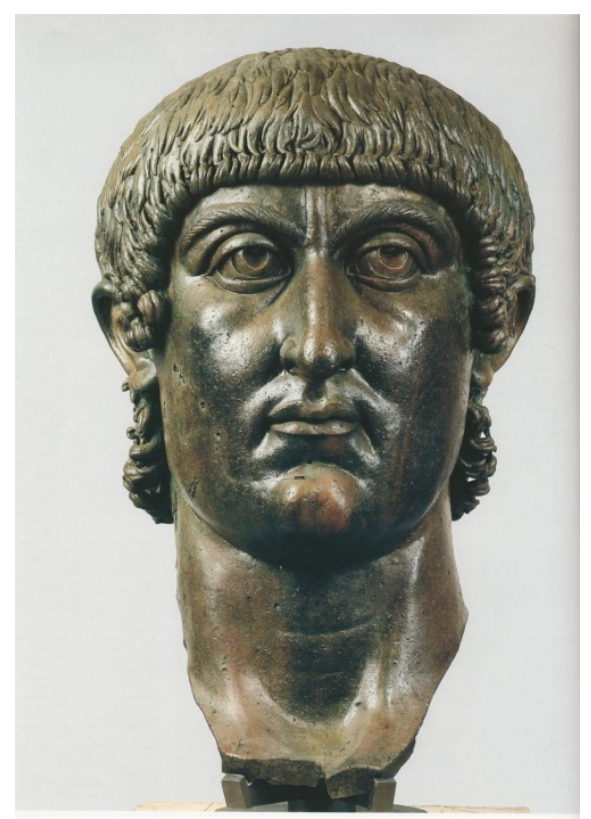

Fig. 14 - Testa colossale in bronzo di Costantino, Roma, Musei Capitolini (da: Ensoli 2000, 72).

dal colosso bronzeo di Costantino, oggi nei Musei Capitolini di Roma (Inv. 1072. FIG. 14). ${ }^{37}$ Quest'ultimo, da datare probabilmente intorno al 340, ovvero qualche anno dopo la morte dell'imperatore (337), lo presenta divinizzato, dopo esser stato accolto da Dio, come figurato nei solidi d'oro coniati dalla zecca di Costantinopoli per Costantino divinizzato, presentandolo nell'immagine del dio Solare (337). Tali monete sono descritte da Eusebio (Vita di Costantino, IV, lxxiii): " "sul recto raffiguravano il sovrano benedetto con il capo coperto da un velo e sul verso lo rappresentavano su una quadriga nelle vesti di un auriga che veniva sollevato in cielo da una mano protesa verso di lui." Illustrando l'imperiale "abbandono della moderazione" ${ }^{\text {, }}$, le monete inscenano nientemeno una vera Ascensione.

Nella Vita di Costantino (IV, xv), ${ }^{40}$ Eusebio racconta anche che l'imperatore "sulle monete d'oro fece incidere la propria effigie (cíkóva) che appariva nell'atto di rivolgere lo sguardo al cielo nel modo in cui si prega Dio con le mani tese verso l'alto. Monete di questo genere circolavano in tutto l'impero romano". Insistendo sullo "sguardo rivolto al cielo" come espressione di preghiera, Eusebio evidentemente si sforza di cristianizzare l'immagine imperiale: "Si potrebbe osservare la grande solennità di tutto ciò considerando che nello stesso palazzo imperiale aveva regolato ogni cosa secondo le modalità di una chiesa di Dio (...)" (ibid., xvii). Tuttavia, neanche il pensiero del rapporto di Costantino col Dio Sole è lontano dal vescovo (Laus Constantini, III, ivv): dopo la morte l'imperatore, dirigendo i cavalli della sua quadriga "sorveglia dall'alto tutta la terra, come lo fa il sole (....." ${ }^{\text {41 }}$ Fino all'ultimo, dunque, era viva l'assimilazione di Costantino al

37. Delbrueck 1933, 139-144, pls. 52-54; Ensoli 2000, 71-81 (tavv. a colori, 72-75).

38. Franco 2009, 426, 427.

39. Parisi Presicce 2005, 138.
40. Franco 2009, 360, 361.

41. LC, III.v; Maraval 2001, 95; “All’unico giogo della sua quadriga reale" Costantino aveva attaccato "i quattro molto valorosi Cesari." 
Sol Invictus, concetto espresso già dallo splendido multiplo d'oro coniato nel 313 dalla zecca di Ticinum (Pavia): al diritto, l'INVICTVS CONSTANTINVS non solo porta uno scudo ornato di un rilievo della quadriga del Sole fiancheggiata dalla Terra e dall'Oceano ma vi è persino ritrattato come vero fratello gemello del dio Sole. ${ }^{42}$

Verso la fine del suo racconto della vita di Costantino - "l'amico di Cristo-Logos" , "l'immagine vivente del Logos, il Figlio di Dio" - l'episcopo di Cesarea $\left(V C\right.$, IV, 1xix, 1-2) ${ }^{43}$ informa che dopo la sua morte, i Romani "celebravano l'imperatore benedetto e caro a Dio", "e non si limitavano a manifestare ciò a gran voce, ma si spingevano a rendere onore all' Imperatore morto anche nei fatti, mediante la dedica dei suoi ritratti come se fosse ancora vivo, riproducendo in dipinti a colori l'immagine del cielo e rappresentando l'imperatore che riposava nelle regioni eteree al disopra della volta celeste". Il ritratto dell'imperatore era una imago sacra ${ }^{44}$ e, eseguita in pittura, altra non era che una icona, qualificazione che Eusebio stesso usa quando racconta che Costantino "vietò per legge che nei templi pagani si dedicassero icone a sua immagine affinché neppure essa si macchiasse dell'errore dei culti che erano stati ripudiati. ${ }^{, 45}$ Dipinta nello stile calligrafico suggerito dal piatto di San Pietroburgo, l'icona imperiale dai tratti della testa di bronzo avrebbe dovuto imprimersi nella mente dello spettatore.

Il colossale ritratto di bronzo, originariamente dorato, è da considerare il principale esempio

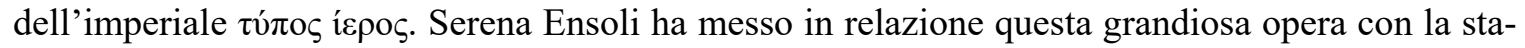
tua di Costantino-Sol, egualmente grandiosa e di bronzo dorato, oggi perduta ma che fino al Medioevo incoronava trionfalmente la colonna porfiretica nel Foro circolare di Costantinopoli. Eretto in relazione alla dedica (l'11 maggio 330) della nuova capitale di Costantino, il monumento ne simbolizzava il centro sacro. La Tabula Peutingeriana, copia medievale del XII o XIII secolo di un rotolo geografico con la descrizione pittorica del mondo antico, probabilmente del IV secolo, su una vignetta dipinta ci mostra Costantinopolis rappresentata da sua Tyche e dalla colonna sormontata da suo fondatore ${ }^{46}$. La statua, rapidamente schizzata, è nuda e porta gli attributi citati dalle fonti, lancia e globo (senza Victoriola), ma manca il nimbo a raggi solari. ${ }^{47}$ In altre rappresentazioni, Costantino-Sole probabilmente indossava l'abito del Dio solare, come l'imperatore di una statuetta del IV-V secolo, trovata in Jutlandia: la testa è coronata di un nimbo a 12 raggi arricchito di un gioiello, e l'imperatore indossa una tunica lunga fino ai piedi e una clamide fermata sulla spalla destra con una fibbia (FIG. 15). ${ }^{48}$

Indipendentemente dall'esattezza o no dell'ipotesi di Serena Ensoli della relazione formale tra il ritratto di Costantino dei Musei Capitolini e quello della colonna a Costantinopoli, credo che il punto di contatto tra la fisionomia imperiale e quella cristiana rappresentata dall'affresco di Cristo nel Tempietto sia stata proprio la statua di autoesaltazione che Costantino fece collocare sulla colonna porfiretica - statua dell'imperatore-Sole che, elevata a quasi 40 metri sopra i comuni mortali, diffondeva la divina claritas sopra la nuova città e la sua popolazione - $\mathrm{o}$, come scrive nel se-

42. Donati, Gentili 2005, 237, no. 54 .

43. Franco 2009, 422, 423.

44. Kruse 1934, 27-39, 49-50; Berger 1981, 26-31.

45. VC, IV.xvi, Franco 2009, 360, 361.

46. Codex Vindobonensis 324. Bosio 1983, 87-88, 149-162, fig. 22, pl. Segmentum VIII. Cf. Harley, Woodward (eds.) $1987,238$.
47. Cf. Bardill, 2012, figs. 17-19 ; Ousterhout 2014, fig. 16.

48. Copenaghen, Nationalmuseum, inv. 8040 (h. 49.7 $\mathrm{cm}$, con gambe/piedi perduti circa $60 \mathrm{~cm})$, Fleischer, Hjort, Bøgh Rasmussen (ed.) 1996, n 10, 46-47 (B. Kiilerich), "Constantinople (?), c. 400." 


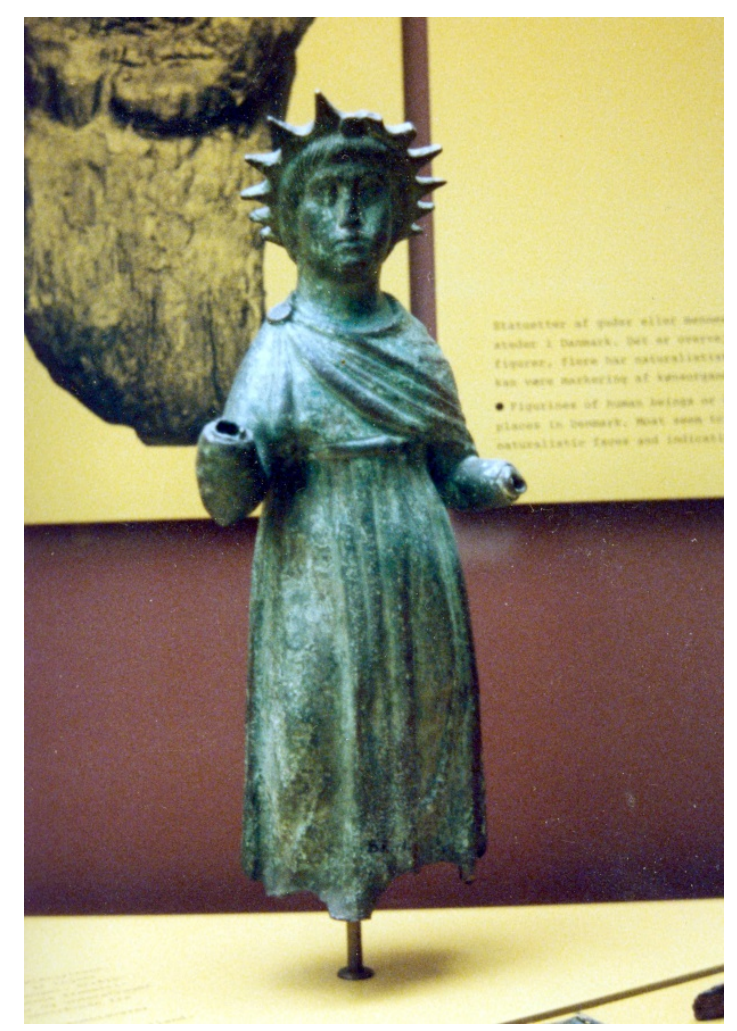

FIG. 15 - Statuetta in bronzo di Costantino-Sol (?), Copenaghen, Nationalmuseum (photo: B. Kiilerich).

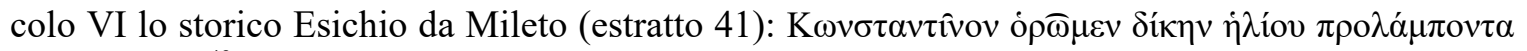

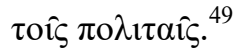

Filostorgio, autore di una Storia Ecclesiastica scritta nella prima parte del V secolo, ci fa sape-

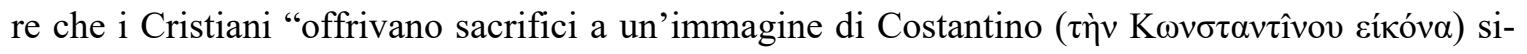
tuata su una colonna di porfido, l'onoravano con lampe accese e incenso e facevano voti alla statua come a Dio ( $\dot{\omega} \theta \varepsilon \tilde{\omega}) " .{ }^{50} \mathrm{Ma}$, a proposito della cristianizzazione dell'immagine imperiale, sono ancora più significative l'erezione e la sistemazione della chiesa dell'Apostoleion. Fatta costruire da Costantino con pianta a croce, "egli aveva tenuto quel luogo in serbo per sé (...), provvedendo, con un impeto di fede straordinaria, che le sue spoglie, dopo la sua dipartita, partecipassero del nome degli Apostoli (...). Pertanto ordinò che in quel santuario si celebrassero anche le funzioni e vi fece sistemare un altare proprio nel mezzo. Nello stesso luogo inoltre, dopo aver fatto innalzare

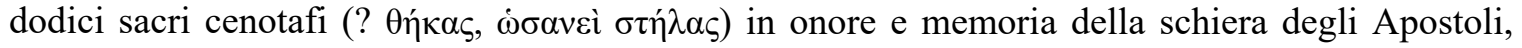
fece collocare esattamente nel centro la propria tomba, su ciascun lato della quale erano disposti sei dei monumenti dedicati agli Apostoli." (Eusebio, Vita di Costantino, IV, lx, 2-3). ${ }^{51}$ D'accordo con Gilbert Dagron, si può concludere con certezza che Costantino tiene effettivamente il posto di Cristo e, sempre con Dagron, in tutta fiducia concludere che a Costantinopoli l'imperatore vittorioso si è sostituito al Cristo risorto di Gerusalemme. ${ }^{52}$

49. Preger 1901, 17. Per il senso religioso della leggenda Claritas Reipvblicae delle monete costantiniane con l'immagine del Sol, si veda Alföldi 1964, 5-16, pl. 3.13.

50. Epitome II.17; Bidez $1972^{2}$; traduzione inglese, Amidon
2007.

51. Franco 2009, 410-413.

52. Dagron 1984, 405-409. 


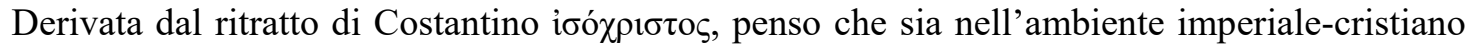
di Costantinopoli del IV secolo che fu creata l'immagine di Cristo del typos hieros dipinta nella lunetta occidentale del Tempietto - forse in relazione alla dedica del 9 aprile 370 del mausoleo imperiale dell'Apostoleion ricostruito. Il rapporto con la tradizione iconografica radicata nell'arte imperiale del secolo IV si conferma dagli scettri coronati da una globetta portati da Michele e Gabriele nelle due lunette e nel mosaico di Kiti (supra, p. 87): questa insegna si vede nella mano di Costantino già nel $315 .^{53}$

Tuttavia, per rafforzare l'idea che l'immagine del Cristo-Luce della lunetta occidentale del Tempietto rappresenti l'adozione ecclesiale della fisionomia del divino Costantino-Sol della colonna porfiretica, occorre lasciare Costantinopoli e spostarsi nel palazzo imperiale di Salonicco, costruito dal tetrarca Galerio (Cesare da 293, Augusto da 305, morto nel 311). La costruzione insigne del palazzo è la grande Rotonda. Situata nella parte settentrionale del complesso imperiale, l'edificio era collegato all'arco di trionfo di Galerio da una maestosa strada porticata. Infatti, trovandosi sull'asse principale sud-nord dell'intero complesso palatino, la Rotonda, la strada e l'arco ne fanno parte integrante tanto dal punto di vista topografico che architettonico. La Rotonda, destinata probabilmente a servire da tempio di culto imperiale, alla morte di Galerio rimase incompiuta, la vasta cupola lasciata a metà. ${ }^{54}$

In un secondo tempo, la costruzione della cupola fu terminata e la Rotonda trasformata in chiesa, con l'aggiunta di presbiterio, abside e ambulacro. Con un diametro di 54 metri, la Rotonda-chiesa era uno dei più grandi edifici cristiani di culto. È da sottolineare, che l'ingresso principale si trovava sempre a sud, come nell'epoca di Galerio, cioè nell'asse centrale nord-sud del complesso imperiale: anche dopo la trasformazione in chiesa, la Rotonda era legata al palazzo. Iniziata da Galerio, da Lattanzio considerato il più crudele persecutore dei cristiani, la Rotonda venne ricostruita e decorata per svolgere la funzione di chiesa palatina - enorme predecessore imperiale della modesta cappella regia di Cividale. La datazione del monumento è discussa. Però, secondo il mio parere, l'iniziativa di trasformare la costruzione di Galerio in chiesa palatina e di decorarla con mosaici sia di attribuire all'imperatore Teodosio I che, dopo essere stato elevato alla dignità di co-imperatore il 19 gennaio 379 a Sirmium, per quasi due anni ebbe sede ufficiale a Tessalonica. ${ }^{55}$ Se iniziata in questo periodo, la trasformazione della Rotonda in chiesa sarà stata verosimilmente ultimata prima del secondo prolungato soggiorno di Teodosio nel palazzo tessalonicense, negli anni 387-388, quando accolse Valentiniano II - fuggitivo dall'Occidente insieme alla madre Giustina e alla sorella Galla, prossima consorte di Teodosio - e celebrò i solenni decennali imperiali (il 19 gennaio 388).

Nella cupola della Rotonda splendevano mosaici a sfondo d'oro e d'argento, disposti in tre zone sovrapposte. In relazione alla genesi dell'immagine di Cristo del Tempietto è d'interesse la zona superiore, e in particolare gli angeli volanti che portano il medaglione con Cristo sullo zenit della cupola. ${ }^{56}$ La testa di uno degli angeli è andata perduta. Delle tre teste conservate, due mostrano facce belle, giovanili, caratterizzate da lineamenti 'angelici', quasi femminei. I paralleli più

53. RIC VII, 1966, 36. Trovo ricercato il dubbio sulla genuinità espresso da Arslan (Donati, Gentili 2012, 199-200). Altro esemplare dei tre medaglioni conosciuti, Engemann 2014, fig. 1. Più tardi, lo stesso scettro è portato per esempio dalla personificazione di Costantinopoli su monete e medaglioni coniati in ricordo della dedica della città 1'11 maggio 330 .

54. Torp 1991, 15-16; Theocharidou 1992, 63, fig. 11.

55. Per le date storiche relative a Teodosio I., si consulti Seeck 1919.

56. Torp 2014, fig. 6a, b. 


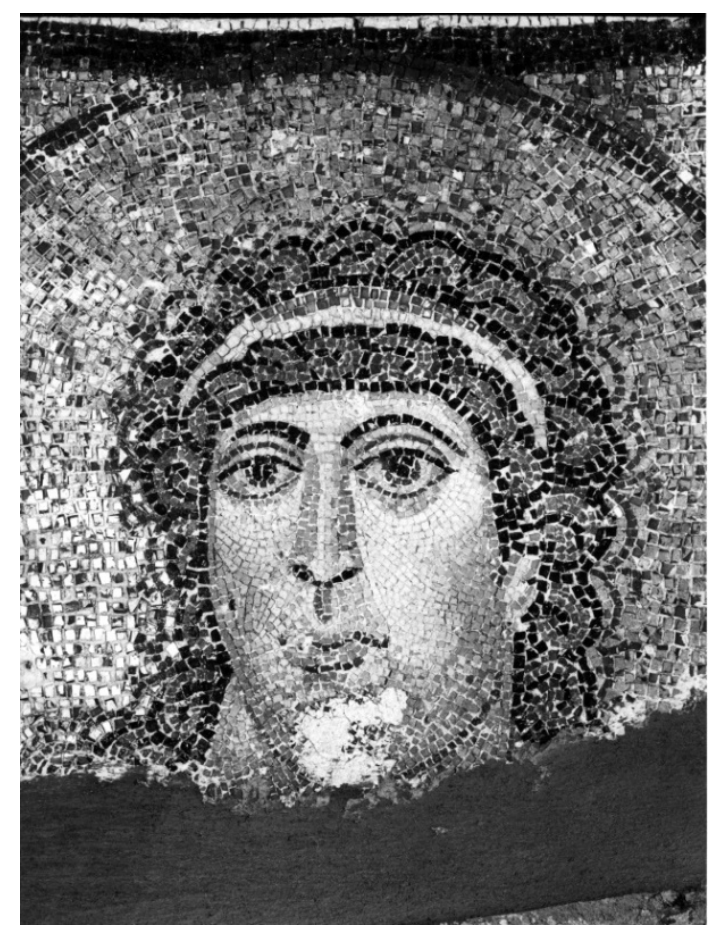

FIG. 16 - Tessalonica, Rotonda, particolare del mosaico della cupola, volto di angelo (photo: H. Torp).

vicini ci sono offerti dagli angeli del cosiddetto Prinzensarkofag, trovato a Istanbul, che fu scolpito negli ultimi decenni del secolo IV, probabilmente da una bottega che lavorava per la famiglia imperiale teodosiana. ${ }^{57}$ Sia dal punto di vista tecnico che iconografico e stilistico-formale penso che i mosaici della Rotonda si debbano similmente spiegare come l'opera di una bottega composta da pittori e mosaicisti il cui capo-bottega e i maestri principali provenivano da Costantinopoli. $^{58}$

Il terzo angelo (FIG. 16) è di tipo del tutto diverso, soprattutto per l'aspetto spiccatamente maschile. La faccia, meno molle, più matura, si distingue specialmente per la robustezza della parte inferiore del viso che, con il mento forte, è di una pesantezza straordinaria, inattesa, per una faccia angelica. La bocca è piena, la fronte larga, ma sorprendentemente bassa; i ricci della frangia sono disposti quasi in linea retta - in chiaro contrasto con la frangia ricciuta degli altri due esseri celesti. Plausibilmente, il mosaico distrutto del quarto angelo aderiva alla stessa, distinta iconografia. Comunque sia, nel terzo angelo il tipo rappresentato dagli angeli gemelli 'effeminati' è stato profondamente modificato, anzi trasformato, sotto l'influenza di un'affermata formula facciale, brutalmente maschile: l'imperiale typos hieros del IV secolo, lo stereotipo fisionomico costantiniano.

Riassumendo nozioni filosofiche e teologiche tradizionali, intorno all'anno 500 lo PseudoDionysio nel suo trattato De coelesti hierarchia, III.2, spiega: La gerarchia, che porta in se stessa il segno di Dio, causa i suoi membri di essere immagini di Dio sotto ogni rapporto, di essere specchi chiari e senza taccia riflettendo lo splendore della luce primordiale, infatti, di Dio lui stesso. ${ }^{59}$ Perciò, essendo gli angeli "specchi chiari” che riflettono Dio, il miglior modo di spiegare l'impe-

57. Kiilerich 2002.

58. Torp 2014.
59. Denys 1'Aréopagite 1958 (= Sources Chrétiennes 58), 87-88, cf. 209, index rerum, s.v. miroir. 
FIg. 17 - Tessalonica, Rotonda, disegno preparatorio per il mosaico di Cristo (disegno S. Sass).

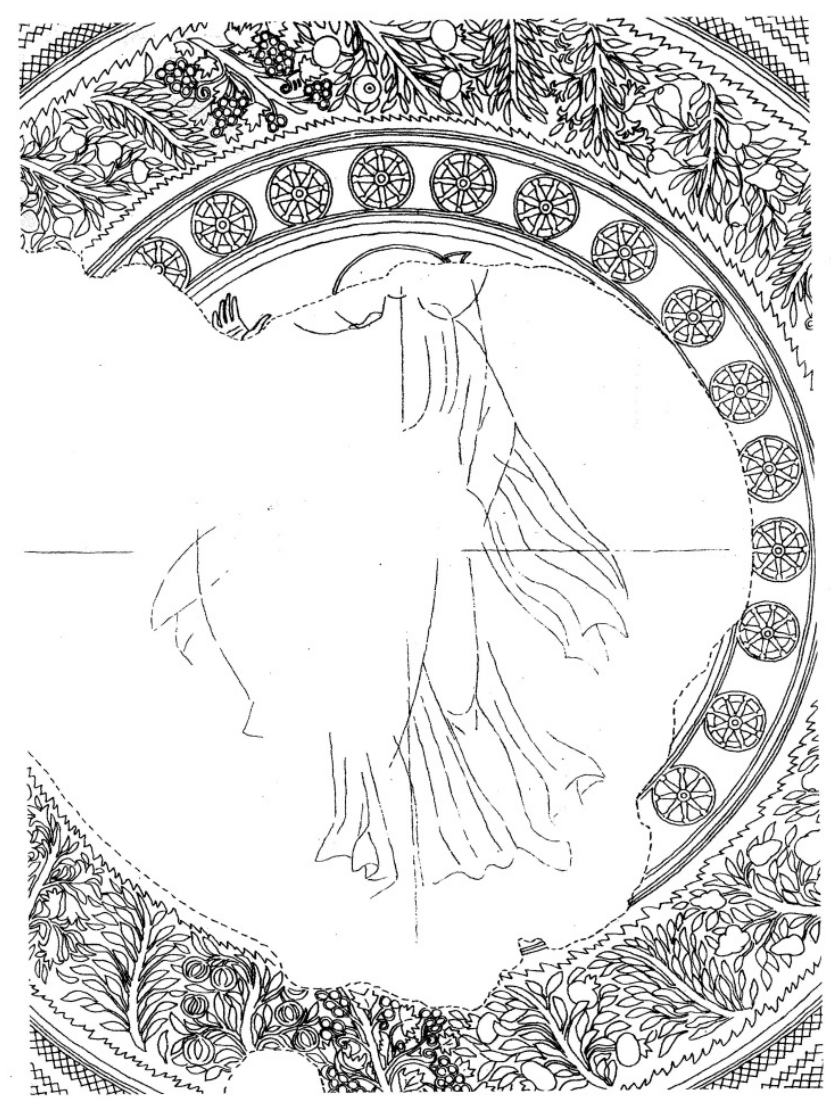

riale typos hieros della faccia del terzo angelo dev'essere appunto di supporre che riflette il viso del Cristo originariamente rappresentato nel medaglione - tipo iconografico alla cui genesi riconosco l'apparizione (nel 310) a Costantino di Apollo-Sol alla somiglianza dell'imperatore stesso. ${ }^{60}$

Se il mosaico del Cristo è quasi del tutto perduto, ne resta invece il disegno preparatorio per il mosaico, tracciato in nero sulla muratura (FIG. 17) ${ }^{61}$ : una figura in piedi, vestito non del solito pallio, ma di un mantello svolazzante, fermato sulla spalla destra, cioè la clamide, e sotto di essa, di un'ampia tunica, lunga fino ai piedi. È l'abito consueto del dio Sole; similmente, la mano destra sollevata a palma aperta del Cristo delineato nel disegno preparatorio è il gesto caratteristico dello stesso dio. Infatti, il gesto e il mantello liberamente svolazzante su ambedue i lati della figura evocano le rappresentazioni caratteristiche del Dio Sole attraversando in piedi sulla sua quadriga la volta celeste - formula iconografica tradizionale che, al più tardi verso l'inizio del IV secolo, fu adottata per esprimere l'onnipotenza cosmica del Signore risuscitato ${ }^{62}$.

La parte superiore del viso è coperta da un frammento di mosaico, ma il disegno ci fa intuire un mento e un collo larghi e forti. Senza dubbio, quindi, similmente all'imperatore della statuetta

60. Panégyrique de Constantin (fin juillet 310), ed.e trad. Galletier, tome II, vii, 1953, 52 (testo 4).
61. Torp 1963, fig. 37; 2014, fig. 6a, b.

62. Miziolek 1991, 31-49, 171-174; figs. 3, 15, 17. 
di Copenaghen, il Cristo rispecchiato nel terzo angelo era presentato nell'immagine tipica del dio Sol. Sulla statuetta come nel disegno preparatorio del mosaico, il chitone lungo fino ai piedi, e la clamide, che è annodata con una fibbia sulla spalla destra e copre la parte superiore del braccio sinistro, costituiscono il completo costume ideale del Sol. La testa della statuetta, poi, illustra il rapporto iconografico generale imperatore-Sol / Cristo-Sol. L'acconciatura, di tipo imperiale (introdotta da Costantino) è diversa da quella ideale a capelli lunghi di Cristo; i lineamenti fisionomici, la fronte bassa, gli occhi e il naso grandi e le guance piene, il mento prominente e il collo largo, invece, definiscono un viso imparentato al vultus divinus di Cristo del Tempietto e dell'angelo Cristo-mimetico dei mosaici della Rotonda.

L'origine, la rapida diffusione, tanto in Occidente quanto in Oriente, e il continuo mantenersi del significato (di tenore giovanneo) dell'immagine di Cristo della lunetta ovest suggerisce che, analogamente alla Hodighitria della lunetta nord, siamo di fronte a una rappresentazione di un particolare stato negli ambienti alti di Costantinopoli. Infatti, l'affresco, dipinto non nel presbiterio ma nell'aula, sopra la porta occidentale che lega il Tempietto e la residenza del gastaldius regis, fa pensare all'immagine di Cristo rappresentata nella lunetta della Chalké a Costantinopoli, la Porta di Bronzo del palazzo imperiale. Però, nell'avorio dell'adventus reliquiarum conservato nel Bischöfliches Dom- und Diözesanmuseum di Treviri, l'unica, relativamente sicura rappresentazione della Chalké, il rilievo della lunetta ci mostra un Cristo sfortunatamente (!) con la barba. ${ }^{63}$ La datazione del rilievo è controversa. Dietro alle divergenti interpretazioni stilistiche (e storiche) dell'avorio sta il fatto che non esiste, o non si conosce, un indiscutibile parallelo iconografico o/e stilistico con la placca. Questo fatto è stato già messo in evidenza da S. Spain, cui siamo debitori dell'esame più dettagliato dello stile del rilievo e che, su criteri formali (e delle insegne imperiali), propende ad assegnarlo alla fine del VI o all'inizio del VII secolo. ${ }^{64}$ Negli ultimi decenni parecchi autori si sono pronunciati in favore di una datazione post-iconoclastica dell'avorio - supposizione indimostrabile che avrebbe fatto proprio il mio gioco, perché in tal caso avrei potuto ipotizzare un Cristo-Chalké pre-iconoclastico del tipo del Cristo-Tempietto. ${ }^{65}$ Non trovo però ammissibile una datazione oltre il VII secolo: malgrado le attuali congetture contrarie, l'avorio di Treviri, a mia convinzione, mostra proprio l'immagine di Cristo che venne rimossa da Leone III all'inizio della iconoclastia. Di come si possa essere presentato un eventuale, più antico Cristo-Chalké non sappiamo nulla. In ogni modo, per lo studio dell'affresco sopra la porta principale del Tempietto, l'avorio di Treviri è di poco aiuto.

Al brano della Vita di Costantino, sopra citato (p. 84), Eusebio aggiunge (IV, xv,2-xvi), "anche nelle residenze imperiali di alcuni città, nelle immagini poste alla sommità delle porte di ingresso egli era raffigurato in piedi nell'atto di guardare il cielo con le mani tese, nell'atteggiamento di chi prega. E questo era il modo in cui era solito farsi rappresentare anche in pittura". È pensabile quindi che il Cristo-Sol sia stata rappresentato sopra altre porte importanti della città. Mi pare più probabile, però, che alla base della scelta dell'immagine del Cristo-Sol, del tutto particolare, si trovino riflessioni simili a quelle sopra suggerite a proposito della Hodighitria: la ne-

63. Delbrueck 1929, I, 261-269; II, pl. 67, “Cpl. 7Jh.?”.

64. Spain 1977.

65. Cf., per esempio, Brubaker, Haldon 2011, 128-135. Secondo Niewöhner 2014, 261 (abstract), l'iconografia dell'avorio "may depict a historical event in its real set- ting: Empress Irene renovates the church of St Euphemia in front of the Hippodrome in 796, shortly after having put up the bust of Christ on the Chalke Gate." 
cessità di invocare soccorso divino per i fondatori reali (p. 81): prima di chiunque altro, a chi affidare la sorte del Regno se non a Cristo-Sol costantiniano? Nella sezione dell'epigrafe dedicatoria che percorre la parete sud del presbiterio (FIG. 5), un esametro della prima riga, (...) IM (...) V (...) V (...) I VIRGO SALVTEM, sembra accennare alla Vergine presente nell'immagine della lunetta nord. ${ }^{66}$ L'invocazione della seconda riga, poi, è rivolta al Figlio, rappresentato nella lunetta occidentale (FIG. 2, 12): Cristo, sovvieni alle preghiere del popolo. "(C)ome a sottolineare un significato o una risonanza particolari" ${ }^{67}$, la formula centrale dell'invocazione è inserita tra due hederae distinguentes:

\section{$\checkmark$ XPE FAVE VOTIS $\vee$}

\section{Epilogo}

Il Tempietto di Cividale costituisce la pietra angolare della costruzione politico-nazionale dell'Italia Langobardorum, Sito del Patrimonio Mondiale UNESCO dal $2011 .^{68}$ Ma si presenta anche, e forse soprattutto, come il punto d'intersezione del decumanus maximus est-ovest et del cardo maximus sud-nord della storia dell'arte altomedievale di Bisanzio e dell'Occidente. Da una parte i soldati martiri, la Vergine Hodighitria e il Cristo Luce ci hanno permesso di aprire delle prospettive sull'arte bizantina pre-iconoclasta, dall'altra di esemplificare la benefica influenza bizantina sull'arte dell'Occidente intorno alla metà del secolo VIII e, quindi, sulla nascente arte carolingia.

Un'osservazione finale. Il Tempietto è un monumento mediterraneo dell'Italia longobarda. Perciò, meglio di qualsiasi documento o altra opera, l'architettura e le decorazioni - particolarmente forse l'iscrizione dedicatoria in esametri latini e il meditato impiego di spolia romani nel presbiterio (due blocchi di trabeazione) - illustrano come la classe dirigente di questa Gente di origine nordico-barbarica mirava ad assimilarsi, anzi, a unirsi alla tradizionale cultura italomediterranea.

Hjalmar Torp

Dokkeveien 4

N-5007 Bergen

66. Tavano 1990, 80-81.

67. Cernuschi 2002, 166. Cf. Tavano 1990, 81 .
68. Per i Beni compresi nel Sito, si veda AA.VV. Dossier di Candidatura. Italia Langobardorum. Centri di Potere e di Culto (568-774 D. C.), 2008, 62-163. 


\section{BIBLIOGRAFIA}

AA.VV. 2008: Italia Langobardorum. Centri di potere e di culto (568-774 d.C.), UNESCO World Heritage List. Dossier di Candidatura, Spoleto.

Alföldi, M.-R. 1964: "Die Sol Comes-Münze von Jahre 325: Neues zur Bekehrung Constantins", in Mullus. Festschrift für Theodor Klauser, JbAC, Erg.B. 1, Münster, 10-16.

Amidon, P.R. 2007: Philostorgius: Church History, AtlantaLeiden.

Bakirtzis, Ch., E. Kourkoutidou-Nikolaïdou, C. Mavropoulou-Tsioumi, 2012: Mosaics of Thessaloniki 4th to 14th Century, Athens.

Bardill, J. 2012: Constantine, Divine Emperor of the Christian Golden Age, Cambridge.

Belting, H. 1968: Studien zur beneventanischen Malerei, Wiesbaden.

Berger, P.M. 1981: The Insignia of the Notitia Dignitatum, New York \& London.

Bertelli, C. 1961: "La Madonna del Pantheon", BdA 46, 2432.

Bidez 1972²: Philostorgius, ed. J. Bidez (1913), $2^{\text {a }}$ ed. a.c.d. F. Winkelmann, Berlin.

Bosio, L. 1983: La Tabula Peutingeriana. Una descrizione pittorica del mondo antico, Rimini.

Brubaker, L. 2012: Inventing Byzantine Iconoclasm, Bristol.

Brubaker, L. \& J. Haldon, 2011: Byzantium in the Iconoclast Era. c. 680-850. A History, Cambridge.

Casadio, P., Perusini, T., Spadea, P. 1996: "Zur Stuckdekoration des "Tempietto Longobardo" in Cividale: Technische und Naturwissenschaftliche Untersuchungsergebnisse", in Stuck des frühen und hohen Mittelalters, 37-51.

Casirani, M. 2002: "La gastaldaga di Cividale: stato delle conoscenze sulle sedi del potere regio nell'Italia longobarda", in Lusuardi Siena 2002, 61-88.

Casirani, M., Cernuschi, S., Codini, L. 2002: "Dati per una riconsiderazione del Tempietto longobardo", in Lusuardi Siena 2002, 107-159.

Cernuschi, S. 2002: "L'iscrizione dipinta del Tempietto", in Lusuardi Siena 2002, 161-174.

Chatzidakis, N. 1994: Byzantine Mosaics, Athens.

Chinellato, L. 2010: "L'altare di Ratchis", in V. Pace, ed., L'VIII secolo: un secolo inquieto, Cividale del Friuli, 8391.

Dagron, G. 1984: Naissance d'une capitale: Constantinople et ses institutions de 330 á 451, Paris.

Degani, A. $1990^{2}$ : Il Tempietto Longobardo di Cividale, Bottenico di Moimacco. de Grüneisen, W. 1911: Sainte Marie Antique, Roma.

Delbrueck, R. 1929: Die Consulardiptychen, Berlin.

Delbrueck, R. 1933: Spätantike Kaiserporträts von Constantinus Magnus bis zum Ende des Westreichs, BerlinLeipzig.

Denys l'Aréopagite 1958: La hiérarchie céleste (Sources Chrétiennes 58), introd. R. Roques, trad. e note M. De Gandillac, Paris.

Dölger, F.J. 1925: Sol Salutis. Gebet und Gesang im christlichen Altertum, Münster.

Donati, A, \& G. Gentili, 2005 (eds.): Costantino il grande. La civiltà antica al bivio tra Occidente e Oriente, Cinisello Balsamo.

Elbern, V.H. 1970: "Neue Aspekte frühmittelalterlicher Skulptur in Gallien," Kolloquium über Spätantike und frühmittelalterliche Skulptur, Mainz, 13-24.

Engemann, J. 2014: Römische Kunst in Spätantike und frühem Christentum bis Justinian, Mainz.

Ensoli, S. 2000: "I colossi di bronzo a Roma in età tardoantica: dal Colosso di Nerone al Colosso di Costantino", in S. Ensoli \& E. La Rocca, eds., Aurea Roma. Dalla città pagana alla città cristiana, Roma, 66-90.

Eusèbe 2001: Eusèbe de Césarée. La théologie politique de L'Empire chrétien. Louanges de Constantin. Introd., trad., P. Maraval, Paris.

Eusebio 2009: Eusebio di Cesarea, Vita di Costantino, a cura di Laura Franco, Milano.

Fleischer, J., Ø. Hjort, M.B. Rasmussen (ed.) 1996: Byzantium. Late Antique and Byzantine Art in Scandinavian Collections, Copenhagen.

Franco L., 2009: Eusebio di Cesarea, Vita di Costantino, a cura di Laura Franco, Milano.

Frolow, A. 1963: "Le Christ de la Chalcé", Byzantion 33, 107-120.

Gabrielli, N. 1953: "Le miniature delle omelie di San Gregorio (codice n. CXLVIII della Biblioteca Capitolare vercellese," in Arte del Primo Millenario (Atti del 2. Convegno per lo studio dell'arte dell'Alto Medio Evo, (Pavia 1950), Torino, 301-311.

Galletier, E. 1953: Les panégyriques latins, tome II, Paris

Harley, J.B. \& D. Woodward, (eds.) 1987: Cartography in Prehistoric, Ancient, and Medieval Europe and the Mediterranean (HOC I), Chicago and London.

Hubert, J., Porcher, J. Volbach, W.F. 1967: L'Europe des Invasions, Paris.

Jeremias, G. 1980: Die Holztür der Basilika S. Sabina in Rom, Tübingen. 
Kiilerich, B. 2002: "The Sarigüzel Sarcophagus and Triumphal Themes in Theodosian Art", in G. Koch, ed., Frühchristliche Sarkophage, Marburg 1999. Sarkophag-Studien 2, Mainz, 137-144.

Kiilerich, B. 2007: "Picturing Ideal Beauty: The Saints in the Rotunda at Thessaloniki", Antiquité tardive 15, 321-336.

Kiilerich, B. 2008: "Colour and Context: Reconstructing the Polychromy of the Stucco Saints in the Tempietto Longobardo at Cividale", Arte medievale anno VII, 2008: 2, 9-24.

Kiilerich, B. 2010: "The Rhetoric of Materials in the Tempietto Longobardo at Cividale", in V. Pace, ed., L'VIII secolo: un secolo inquieto, Cividale del Friuli, 93-102.

Koehler, W. 1933: Die Karolingischen Miniaturen I: Die Schule von Tours, vol. 2: Die Bilder, Berlin.

Koehler, W. 1958: Die Karolingischen Miniaturen II: Die Hofschule Karls des Grossen, Berlin.

Kruse, H. 1934: Studien zur offiziellen Geltung des Kaiserbildes im roemischen Reiche, Paderborn.

Lohuisen-Mulder, M. 1990: "Stuccoes in Ravenna, Porec and Cividale of Coptic Manufacture", BABesch 65, 139-156.

L'Orange, H.P. 1953: "L'originaria decorazione del Tempietto Cividalese", in Atti del 2. Congresso internazionale di studi sull'alto medioevo, Spoleto (1952), 95-113.

Lusuardi Siena, S. (ed.) 2002: Cividale Longobarda. Materiali per una rilettura archeologica, Milano.

Lusuardi Siena, S. 2002: "Per una rilettura delle fasi edilizie del Tempietto", in Lusuardi Siena 2002, 205-250.

Miziolek, J. 1991: Sol Verus, Warszaw.

Nelson, R.S. \& K.M. Collins, (ed.) 2006: Holy Image. Hallowed Ground. Icons from Sinai, Los Angeles.

Niewöhner, Ph. 2014: "Historisch-topographische Überlegungen zum Trier Prozessionselfenbein, dem Christusbild an der Chalke, Kaiserin Irenes Triumph im Bilderstreit und der Euphemiakirche am Hippodrom", Millenium 11, 261288.

Nordhagen, P.J. 2001: "The Absent Ruler: Reflections on the Origin of the Byzantine Domed Church and its Pictorial Decoration", ActaAArtHist XV (n.s. 1), 319-336.

Nordhagen, P.J. 2004: "Iconoclasm: Rupture or Interlude? A Reassessment of the Evidence", ActaAArtHist XVIII (n.s. 4), 205-215.

Ousterhout, R. 2014: "The Life and Afterlife of Constantine's Column", JRA 27, 304-326.

Pace, V. 2004: "Immagini sacre a Roma fra VI e VII secolo. In margine al problema "Roma e Bisanzio", ActaAArtHist XVIII (n.s. 4), 139-156.

Pace, V. 2015: “Alla ricerca di un'identità: affreschi, mosaici, tavole dipinte e libri a Roma fra VI e IX secolo", in S. Lucà (ed.), Roma e il suo territorio nel medioevo (Atti del convegno, Roma 2012), Spoleto, 471-498.

Parisi Presicce, C. 2005: "L'abbandono della moderazione. I ritratti di Costantino e della sua progenie", in A. Donati \& G. Gentili, ed., Costantino il grande. La civiltà antica al bivio tra Occidente e Oriente, Cinisello Balsamo, 138-155.

Preger, Th. 1901: Scriptores originum constantinopolitanarum, I (ed. Th. Preger), Leipzig (New York 1975²).

Pseudo-Dionysius 1986: Pseudo-Dionysius Areopagita, Über die himmlische Hierarchie. Über die kirchliche Hierarchie, ed. G. Heil, Stuttgart.

Rugo P. \& O. Rugo, 1990: Il Tempietto Longobardo di Cividale del Friuli, Pordenone.

Rushfort, G.M. 1902: "The Church of S. Maria Antiqua", BSR 1, 1-123.

Seeck O. 1919: Regesten der Kaiser und Päpste für die Jahre 311 bis 476 n.Chr., Stuttgart.

Spain, S. 1977: "The Translation of Relics Ivory, Trier", DOP 31, 279-304.

Tavano, S. 1990: Il Tempietto longobardo di Cividale, Udine.

Theocharidou, K. 1992: "H Rotonda tes Thessalonikis. Nea stoicheia kai aposafeniseis me aforme tes anastelotikes", DeltChrA 4, 1991-92, 57-76.

Torp, H. 1953: "Note sugli affreschi più antichi dell'oratorio di S. Maria in Valle a Cividale", in Atti del 2. Congresso internazionale di studi sull'alto medioevo, Spoleto (1952), 81-93.

Torp, H. 1959: Il problema della decorazione originaria del Tempietto Longobardo di Cividale del Friuli (Quaderni della FACE, XVIII), Udine (download:

https://uib.academia.edu/BenteKiilerich/papers).

Torp, H. 1963: Mosaikkene i St. Georg-Rotunden, Oslo.

Torp, H. 1974: "Der Tempietto in Cividale und seine Ausstattung. Ein Monument der spätlangobardischen Hofkunst", Kolloquium über Spätantike und Frühmittelalterliche Skulptur (1972), Mainz, 1-13.

Torp, H. 1977: Il Tempietto Longobardo di Cividale. L'architettura del Tempietto (ActaAArtHist VII,2), Rome.

Torp, H. 1984: The Integrating System of Proportion in Byzantine Art. An Essay on the Method of the Painters of Holy Images (ActaAArtHist s.a..4 $4^{\circ}, 18$ ).

Torp, H. 1991: "The Date of the Conversion of the Rotunda at Thessaloniki into a Church", Papers from the Norwegian Institute at Athens 1, 13-28.

Torp, H. 1999: "Una Vergine Hodighitria del periodo iconoclastico nel 'Tempietto Longobardo' di Cividale", in A. Cadei, e.a. (eds)., Arte d'Occidente. Temi e metodi. Studi in onore di Angiola Maria Romanini, vol. II, Roma, 583-599.

Torp, H. 2006: Il Tempietto Longobardo. La Cappella Palatina di Cividale, Cividale.

Torp, H. 2009: "Mario Brozzi e il dilemma carolingiolongobardo del Tempietto di Cividale", Forum Iulii XXXIII, 23-30.

Torp, H. 2011: “An Interpretation of the Early Byzantine Martyr Inscriptions in the Mosaics of the Rotunda at Thessaloniki", ActaAArtHist XXIV (n.s. 10), 11-43.

Torp, H. 2014: "La technique des mosaïques de la Rotonde de Thessalonique", Arte medievale, IV. ser, anno IV, 267280 .

Vaj, I. 2002: "Il tempietto di Cividale e gli stucchi omayyadi", in Lusuardi Siena 2002, 175-204.

Volbach, W. F. 1952: Elfenbeinarbeiten der Spätantike und des frühen Mittelalters, Mainz.

Weis, A. 1958: "Ein vorjustinianischer Ikonentypus in S. Maria Antiqua”, Römisches Jahrbuch für Kunstgeschichte $8,19-61$. 
